\title{
High-pressure reaction profiles and activation volumes of 1,3- cyclohexadiene dimerizations computed by the extreme pressure-polarizable continuum model (XP-PCM)
}

\author{
Bo Chen, ${ }^{1,2,{ }^{*}}$ K. N. Houk, ${ }^{3}$ and Roberto Cammi ${ }^{4, *}$ \\ ${ }^{1}$ Donostia International Physics Center, Paseo Manuel de Lardizabal, 4, 20018 Donostia- \\ San Sebastian, Spain \\ ${ }^{2}$ IKERBASQUE, Basque Foundation for Science, Plaza Euskadi 5, 48009 Bilbao, Spain \\ ${ }^{3}$ Department of Chemistry and Biochemistry, University of California, Los Angeles, \\ California 90095, United States \\ ${ }^{4}$ Department of Chemical Science, Life Science and Environmental Sustainability, \\ University of Parma, Viale Parco Area delle Scienze. 17/a, Parma, 43100, Italy \\ bo.chen@dipc.org; roberto.cammi@unipr.it
}

\begin{abstract}
Quantum chemical calculations are reported for the thermal dimerizations of 1,3cyclohexadiene at $1 \mathrm{~atm}$ and high pressures up to $6 \mathrm{GPa}$. Previous experiments [Klärner et al. Angew. Chem. Int. Ed. 1986, 25, 108], based on measured activation energies and activation volumes, suggested concerted mechanisms for the formation of the endo [4+2] cycloadduct and a [6+4]-ene adduct, and stepwise mechanisms for the formation of the exo $[4+2]$ cycloadduct and two [2+2] cycloadducts. Computed activation enthalpies $(\omega \mathrm{B} 97 \mathrm{XD}$, CCSD(T) and SC-NEVPT2) of plausible dimerization pathways at 1 atm agree well with the experiment activation energies and the values from previous calculations [Ess et al. J. Org. Chem. 2008, 73, 7586]. High-pressure reaction profiles, computed by the recently-
\end{abstract}


developed extreme pressure-polarizable continuum model (XP-PCM), show that the reduction of reaction barrier is more profound in concerted reactions than in stepwise reactions, which is rationalized on the basis of the volume profiles of different mechanisms. A clear shift of the transition state towards the reactant by high pressure is revealed for the $[6+4]$-ene reaction by the calculations. The computed activation volumes by XP-PCM agree excellently with the experimental values, confirming the existence of competing mechanisms in the thermal dimerizations of 1,3-cyclohexadiene.

\section{Introduction}

The rates of chemical reactions are usually affected by both temperature and pressure. The effect of temperature on the rate of a chemical reaction is measured as the activation energy ${ }^{1,2}$ For thermally activated reactions (no tunneling 3 involved), the larger the activation energy $E_{a}$, the greater the increase in the reaction rate with temperature. ${ }^{4}$ Activation energies are measured experimentally by treating the temperature dependence of the rate constant using the Arrhenius equation. The activation energy is the slope of the plot of $\ln k$ vs $1 / T, E_{a}=R\left(\frac{\partial \ln k}{\partial(1 / T)}\right)_{p}$, where $k$ is the rate constant, $T$ the temperature, $R$ the ideal gas constant, and $p$ the pressure.

The effect of pressure on the rate of a chemical reaction is measured by the volume of activation (or activation volume) $\Delta V^{\ddagger} .5,6,7,8,9,10,11,12,13,14,15,16,17,18$ The volume of activation is the volume change of a reaction system from the reactant(s) to the transition state. Experimentally, activation volumes are obtained by studying the pressure dependence of the rate constants using $\Delta V^{\ddagger}=\left(\frac{\partial \Delta G^{\ddagger}}{\partial p}\right)_{T}=-R T\left(\frac{\partial \ln k}{\partial p}\right)_{T}$, where $\Delta G^{\ddagger}$ is the Gibbs energy of activation, according to the thermodynamic formulation of the transition state theory. $4,19,20,21,22$

While activation energies are almost always positive, ${ }^{23}$ activation volumes can commonly be both positive and negative. Reactions with negative and positive $\Delta V^{\ddagger}$ are accelerated and decelerated by pressure, respectively. Usually, the signs of activation volumes can be intuitively predicted. For example, in the case of a bond formation reaction 
between two molecules (Figure 1), a negative $\Delta V^{\ddagger}$ is expected since the unimolecular transition state is more compact and hence has a smaller volume than the two separated reactant molecules. The opposite is usually true for bond cleavage reactions.

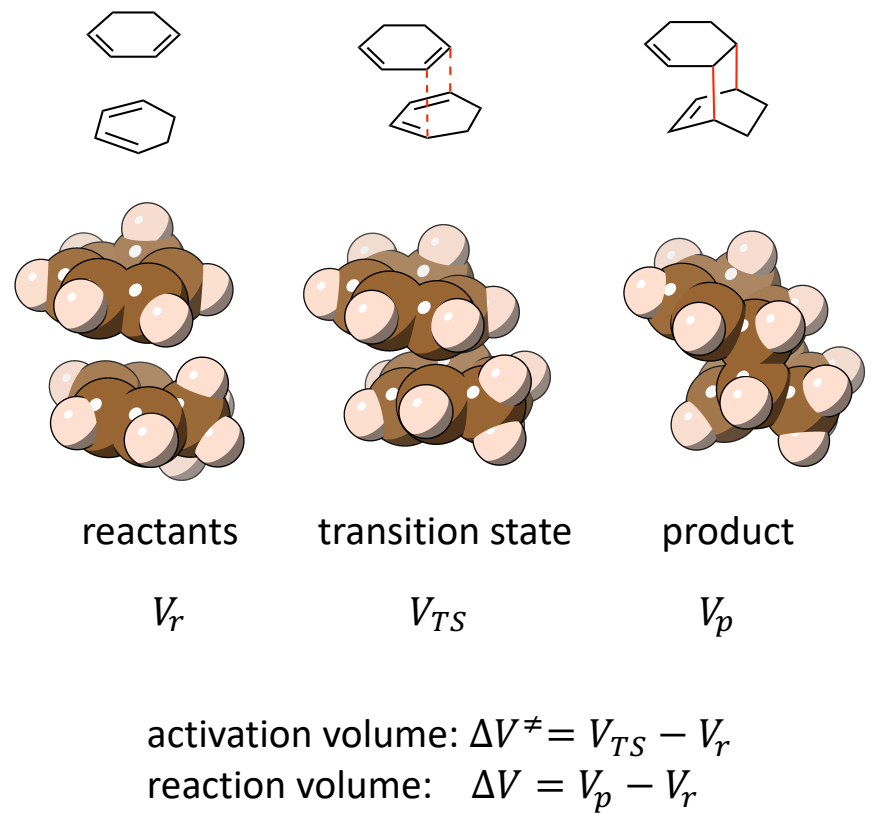

Figure 1. Schematic illustration of the activation volume and reaction volume (both are negative) of a Diels-Alder dimerization of 1,3-cyclohexadiene.

Measurements of $\Delta V^{\ddagger}$ are a valuable tool to distinguish different reaction mechanisms. A notable example is the case of several Diels-Alder reactions whose activation volumes have given evidences in favor of a concerted one-step mechanism over the competing two-step mechanism. ${ }^{24,25}$ Another exemplary case of competing mechanisms is revealed by the illuminating study of the effect of pressure on the thermal dimerization of 1,3-cyclohexadiene by Klärner et al in $1986 .{ }^{26}$

At $1 \mathrm{~atm}$, thermal dimerization of 1,3-cyclohexadiene yielded five reaction products-two [4+2] cycloadducts of the endo and exo configurations, two [2+2] cycloadducts of the syn and anti configurations, and one [6+4]-ene adduct (Figure 2). It was also observed that, under pressure up to $7 \mathrm{kbar}$, the reactions producing all five products 
are accelerated, indicating negative activation volumes for all these reactions. Considering the bond-formation nature of these reactions, the observed acceleration under pressure is not surprising.

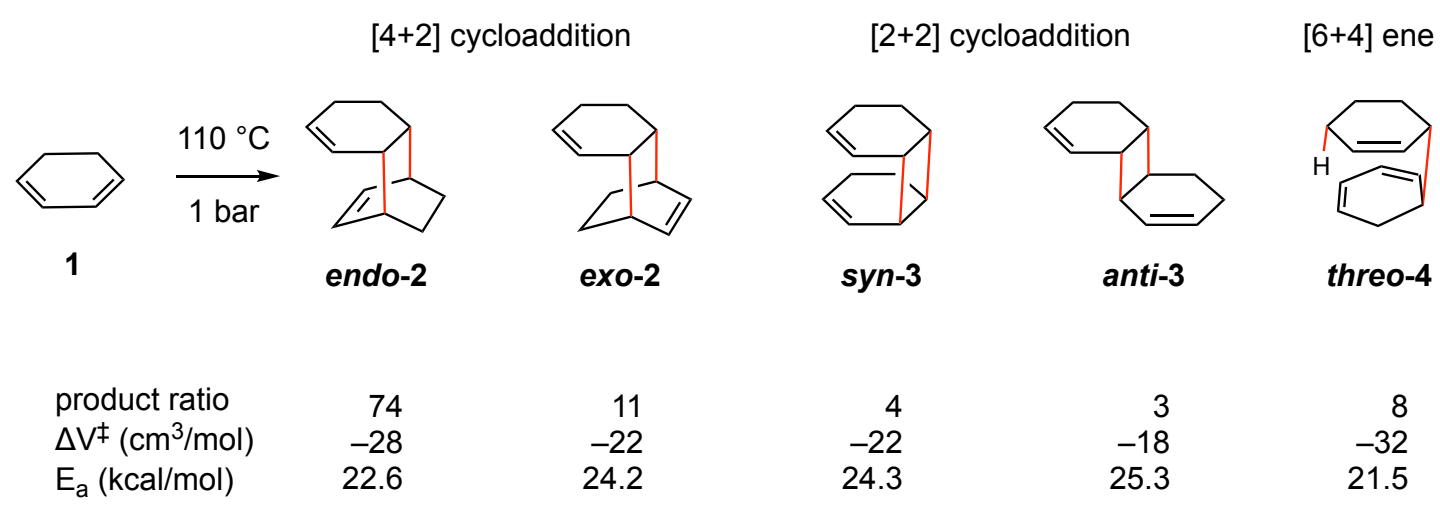

Figure 2. The observed products of thermal dimerizations of 1,3-cyclohexadiene. New bonds are in red. Experimentally measured activation volumes $\left(\Delta V^{\ddagger}\right)$ and activation energies $\left(E_{a}\right)$ are shown.

Apparently, different mechanisms are in operation in this dimerization, for both the symmetry-allowed [4+2] and [6+4] and forbidden [2+2] dimers, according to the Woodward-Hoffmann rules, ${ }^{27}$ were observed. The symmetry-allowed [4+2] cycloadducts and the [6+4]-ene adduct could, in principle, be formed through either a concerted or stepwise mechanism, whereas the symmetry-forbidden [2+2] cycloadducts are usually expected to be formed via a stepwise mechanism under thermal conditions (Figure 3). ${ }^{26,28}$ The main difference between the concerted and stepwise mechanisms, in terms of activation volume, is that two bonds are formed at once in a concerted mechanism and only one bond is initially formed in a stepwise mechanism, so that the concerted transition state (TS) structure is usually more compact than the stepwise TS structure. Therefore, the concerted reaction usually has a more negative activation volume than a stepwise reaction. 


\section{Concerted [4+2]-cycloaddition pathways}

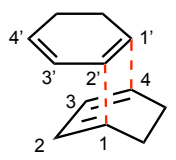

TS-endo-2

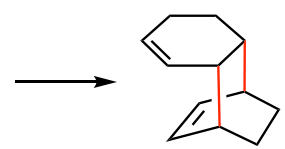

endo-2

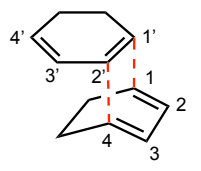

TS-exo-2

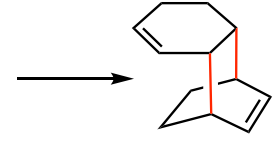

exo-2

Concerted [6+4]-ene pathways

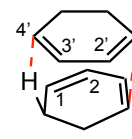

TS-threo-4

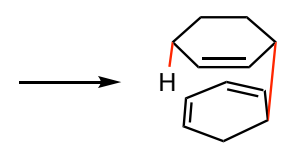

threo-4

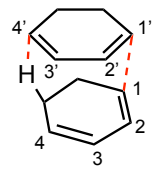

TS-erythro-4

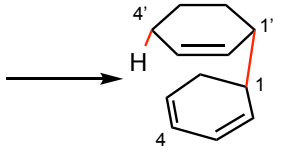

erythro-4 not observed

Concerted [4+2]-ene pathways<smiles>C1=CC(C2CC3CCC2C3)C2CCC1C2</smiles>

TS-5<smiles>C1=CC2CCC3CCC(CC3)C2C1</smiles>

TS-6 or<smiles>C1=CC2CCCC3C=CCC3C(C1)C2</smiles>

TS'-5

or

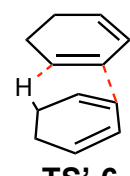

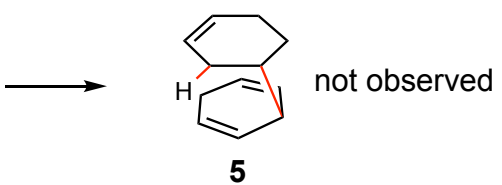

$\longrightarrow$ not observed

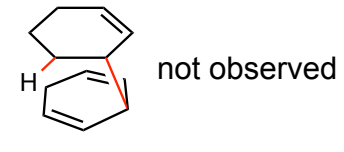

6

\section{Stepwise pathways}
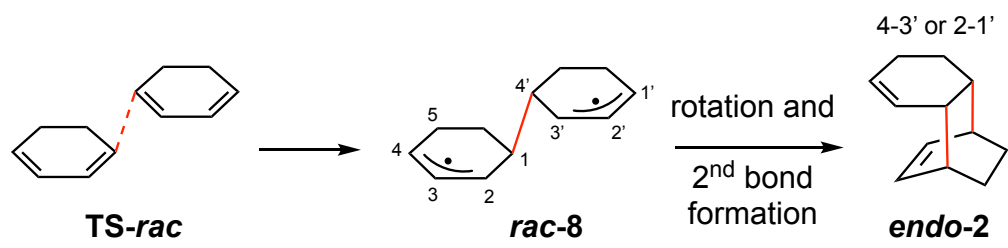

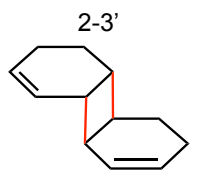

anti-3

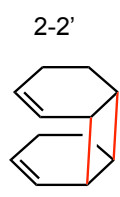

syn-3
5-1' H-transfer

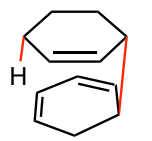

threo-4

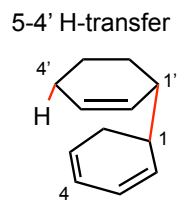

erythro-4

not observed

Figure 3. Plausible concerted and stepwise mechanisms. The ring position numbering of 1,3cyclohexadiene follows a clockwise fashion, with primed and unprimed numbers for different rings. 
Klärner et al. ${ }^{26}$ observed (Figure 2) that the reactions yielding the endo [4+2]cycloadduct and the threo [6+4]-ene adduct were accelerated the most by pressure. Measurements of volumes of activation show that the reaction leading to endo-2 has $\Delta V^{\ddagger}=$ $-28 \mathrm{~cm}^{3} / \mathrm{mol}$ and the reaction leading to threo-4 has $\Delta V^{\ddagger}=-32 \mathrm{~cm}^{3} / \mathrm{mol}$. The reactions leading to the other three observed products have smaller $\Delta V^{\ddagger}$ between -22 and -18 $\mathrm{cm}^{3} / \mathrm{mol}$.

Based on these $\Delta V^{\ddagger}$ data, Klärner et al. ${ }^{26}$ reasoned that $\mathbf{e n d o - 2}$ and $\boldsymbol{t h r e o - 4}$ are most likely formed by concerted mechanisms because their relatively large and negative volumes of activation suggest highly compact TS structures. On the other hand, the other three reactions with less negative activation volumes are more compatible with stepwise mechanisms. Interestingly, the reactions affording the [4+2]-adduct exo-2 and [2+2]adduct syn-3 were measured to have essentially the same activation volume and activation energy, which led Klärner et al. to argue that these two reactions likely follow a common stepwise mechanism.

The measured activation energies $E_{a}$ for the reactions giving the five products in Figure 2 are within $4 \mathrm{kcal} / \mathrm{mol}$. An even smaller difference in $E_{a}$ is seen between the endo and exo [4+2]-cycloadditions, though the former likely follows a concerted mechanism while the latter a stepwise mechanism, based on the measured difference in $\Delta V^{\ddagger} .{ }^{26}$ Interestingly, it seems that the more negative the $\Delta V^{\ddagger}$, the lower the $E_{a}$ of the reaction, though the two quantities do not necessarily have a correlation.

In 2008, Ess et al. calculated the TS structures and reaction pathways for the dimerization of 1,3-cyclohexadiene in the gas-phase. ${ }^{28}$ Calculations at B3LYP, CASPT2 and CBS-QB3 levels show that the computed reaction barriers for the concerted [4+2]cycloadditions, concerted [6+4]-ene reactions, and the stepwise additions (Figure 3 ) are within $5 \mathrm{kcal} / \mathrm{mol}$. This small difference is consistent with the difference in experimental $E_{a}$, confirming the competitive nature of the concerted and stepwise mechanisms. However, the exact energetic order of these TS structures is slightly different between the calculation and experiment. 
The Ess et al. work focused on the activation energies and didn't deal with the activation volumes of the reaction. Theoretical calculations of $\Delta V^{\ddagger}$, especially from firstprinciples, have been a challenge. A simple and intuitive way to compute $\Delta V^{\ddagger}$ is to calculate the difference in van der Waals ( $\mathrm{vdW}$ ) volumes of the molecule at the reactant and transition states. The vdW volume is the volume of interlocking vdW spheres (often with scaled vdW radii) centered on the constituting atoms of the molecule. However, likely due to the negligence of intermolecular interactions and solvent effect, $\Delta V^{\ddagger}$ computed by this method are always too small in magnitude. An empirical packing coefficient was introduced to account for such negligence and to correct the underestimated $\Delta V^{\ddagger}$, computed by this method. ${ }^{15,29}$

A new method for more rigorous $\Delta V^{\ddagger}$ calculations is the recently-developed extreme pressure polarizable continuum model (XP-PCM). ${ }^{30}$ As an extension of the popular polarizable continuum model (PCM) that tackles molecular solution energies at the standard condition of pressure, the XP-PCM allows for quantum chemical calculations of the energy profiles of chemical reactions under pressure. The effect of the pressure is introduced in XP-PCM via a repulsive interaction between the reactive molecular system and the surrounding solvent medium. Within XP-PCM, $\Delta V^{\ddagger}$ can be computed, according to the transition state theory, as the derivative of activation free energy with respect to pressure. The XP-PCM method has been applied to the calculations of the energy profiles of a subset of pericyclic reactions. ${ }^{17,31}$ Interesting phenomena such as a shift of the transition state and a switch of the rate determining step have been discovered by the calculations. ${ }^{17}$ Furthermore, the computed $\Delta V^{\ddagger}$ are in reasonable agreement with experimental value. ${ }^{17}$ Recent work by Fukuda and Nakatani applied the XP-PCM method to a retrocycloaddition. ${ }^{32}$ The necessary details of the physical basis and computational protocol of the XP-PCM method are given in the Computational Methodology section. In additional to reaction profiles, the XP-PCM method has also been applied to the studies of the effect of pressure on a variety of molecular properties, such as equilibrium geometries, ${ }^{33,34,35}$ vibrational frequencies, ${ }^{36,37,38}$ electronic excitation energies. ${ }^{39}$

We note here relevant approaches derived from the mechanochemistry field for high-pressure calculations on molecules and reactions. ${ }^{40,41,42,43}$ Notably, the recent 
GOSTSHYP method from the Stauch group ${ }^{44}$ is capable of calculating activation volumes of reactions. Another approach for simulating high-pressure organic reactions employs a simulation box with periodic boundary condition, filled with solvated reactant molecules. The solvent molecules are explicitly included in the simulation box, in contrast to the implicit solvation approach in the XP-PCM method. Molecular dynamics (MD) simulations or Monte Carlo simulations (an early example by Klärner et al. ${ }^{25}$ ) are performed to obtain reaction profiles at high pressures and activation volumes. Due to the large size of the system using a simulation box, the MD simulations were usually done with force fields, as illustrated in the works from the Weinberg group, ${ }^{45,46,47,48}$ or by a hybrid quantum mechanics/molecular mechanics (QM/MM) approach, as shown in the work by Plotnikov and Martinez ${ }^{49}$ and a recent work by Loco et al. ${ }^{50}$

We now report a thorough consideration of the potential energy surface (PES) of the thermal dimerization 1,3-cyclohexadiene, including [4+2]-ene pathways that were not considered previously. In addition, we report, for the first time, XP-PCM calculations on the activation volumes for the various dimerization reactions of 1,3-cyclohexadiene, offering a new approach for computationally studying the competing mechanisms of this reaction.

\section{Computational Methods}

Gas-phase calculations. Gas-phase geometries were optimized at the $\omega B 97 X D^{51} /$ def2-TZVP ${ }^{52}$ level of theory. Frequency analyses were performed at the same level to verify the optimized structure to be either a minimum or a transition state, and to obtain the zero-point vibrational, thermal, and entropic corrections, necessary in calculating enthalpies and free energies. For open-shell singlets that appear in the stepwise mechanism, broken-symmetry DFT with spin-projection by the Yamaguchi-Houk procedure ${ }^{53}$ was used. On the $\omega$ B97XD/def2-TZVP optimized structures, single-point calculations were performed using the strongly contracted $\mathrm{N}$-electron valence state perturbation theory (SC-NEVPT2) ${ }^{54,55,56}$ and coupled-cluster $\left.\operatorname{CCSD(T)}\right)^{57,58}$ methods with the same def2-TZVP basis set. In the SC-NEVPT2 calculations, a $(4,4)$ active space was used for 
1,3-cyclohexadiene, which comprises the valence $\pi$-type orbitals and the valence $\pi$ electrons in the molecules. An $(8,8)$ active space was used for the transition states, which consists of the same set of $\pi$-type orbitals and $\pi$ electrons of two molecules of 1,3cyclohexadiene; however, some of the $\pi$-type orbitals become more of $\sigma$-type orbitals in the bond formation regions in the transition states. The $\omega \mathrm{B} 97 \mathrm{XD}$ and $\operatorname{CCSD}(\mathrm{T})$ calculations were computed using the Gaussian $16^{59}$ program package. The SC-NEVPT2 calculations were done with the ORCA 4.2.160,61 program package.

High-pressure calculations. High pressure calculations were performed using the XP-PCM method at the $\omega$ B97XD/def2-TZVP level of theory in Gaussian 16 and an in-house Julia script. The XP-PCM is a quantum chemical method aimed to introduce the effects of the pressure on the calculation of the electronic energy $G_{e r}$ of a molecular system in a dense medium via a Pauli exchange-repulsion interaction between the molecular system and the external medium. Such a Pauli-exchange repulsion is motivated by the fact that at high pressure, the reduction of the volume of a dense medium forces the intermolecular distances below the van der Waals contacts, in a domain where the intermolecular interactions are dominated by the Pauli exchange-repulsion. ${ }^{62}$ The increase of the pressure is modeled by simply shrinking the volume $V_{c}$ of the cavity hosting the molecular system so as to increase the overlap between the electron densities of the system and of the external medium (Figure 4). This molecular cavity is built up starting from the envelope of vdW spheres centered on the nuclei of the reactive system and with scaled vdW radii. 


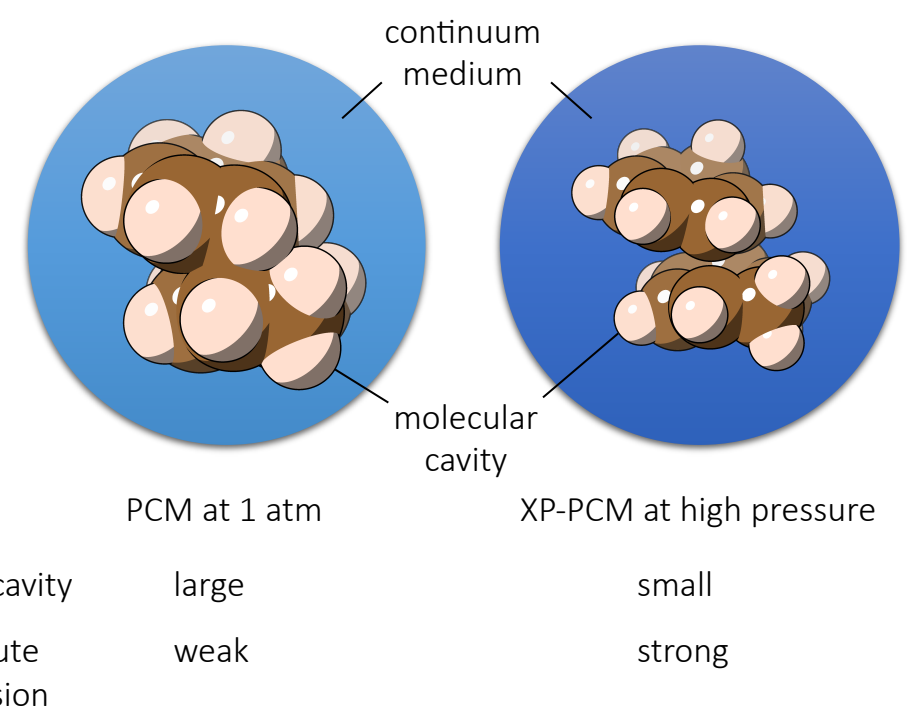

Figure 4. The XP-PCM model as an extension of the PCM model. A molecular system is confined in a molecular-shaped cavity (in this case the vdW cavity) in the external medium (in blue). The combination of a smaller size of the cavity and a stronger solvent-solute Pauli repulsion in XP-PCM models the effect of compression on the molecule.

In studying chemical reactions at high pressure, the effective potential energy for the motion of the nuclei of the reactive system, $G_{t o t}(p)$, corresponds to the electronic energy $G_{e r}(p)$, supplemented by a contribution of the so-called cavitation Gibbs energy that corresponds to the work necessary to create the void cavity hosting the molecular solute at the given condition of pressure $p$ :

$$
G_{t o t}(p)=G_{e r}(p)+G_{c a v}(p) .
$$

This work also includes a configurational entropic contribution from the external medium. ${ }^{63}$ The effective potential energy profile for a reaction at a given pressure $p$ is then determined by computing $G_{t o t}(p)$ for a set of selected structures along a suitable reaction coordinate. According the transition state theory, the activation volume $\Delta V^{\ddagger}$ is determined from the slope of the corresponding activation energy $\Delta G_{t o t}^{\ddagger}(p)$ as a function of the pressure,

$$
\Delta V^{\ddagger}=\Delta G_{t o t}^{\ddagger}(p) / d p
$$


In this work, the effective potential energy profile for a reaction at a given pressure $p$ is then determined by computing $G_{t o t}(p)$. The corresponding activation volume $\Delta V^{\ddagger}$ is computed by the same computational protocol used previously for the study of the effect of pressure on a selection of pericyclic reactions, where reasonable agreement of the computed activation volumes with experiment values was obtained. ${ }^{17}$ We recommend that interested readers consult ref 17 and the SI of the current paper for a detailed tutorial about the protocol. All the XP-PCM calculations are performed for selected structures (reactants, transition state, product) along the gas phase intrinsic reaction coordinates.

\section{Potential energy surface at $1 \mathrm{~atm}$}

Mechanisms considered. As shown in Figure 3, the concerted pathways considered in this paper include [4+2]-cycloadditions, [6+4]-ene reactions, and [4+2]-ene reactions. The [4+2]-ene pathways were not considered in previous calculations by Ess et al. ${ }^{28}$ For the concerted [4+2]-cycloadditions and [6+4]-ene reactions, each of these pathways can generate two stereo isomers as a result of different relative orientations of the two cyclohexadiene rings in the TS structures. These isomers are termed endo and exo for the [4+2] cycloaddition adducts and threo (also could be called rac or $\mathrm{dl}$ ) and erythro (also could be called meso) for the [6+4] ene-adducts.

For concerted [4+2]-ene reactions, two pathways are possible. In each of them, there exist configurational isomers for the TS, for example, TS-5 and TS'-5. However, both of these two TS structures lead to the same dimer (i.e., 5). The configurational isomerism in the TS disappears in the dimer because the bottom ring in the adduct becomes 1,4cyclohexadiene that does not contain any stereocenter.

For stepwise pathways, we considered those beginning with a $\mathrm{C}-\mathrm{C}$ bond formation between two 1,3-hexadiene molecules at the terminus of the diene moieties and generating a diallyl intermediate, $\mathbf{r a c}-\mathbf{8}$ or meso-8. Either of the two intermediates contains two stereocenters, but the $\mathbf{m e s o - 8}$ isomer is achiral due to the presence of an inversion center in the structure. Other stepwise pathways involving bond formation at internal sites of the 
diene moiety are likely to be unfavorable due to the generation of isolated (i.e., not in conjugation with a double bond) radical sites; these pathways are not considered. In the second step, a rotation about the first formed $\mathrm{C}-\mathrm{C}$ bond followed by a radical recombination or a $\mathrm{H}$-transfer leads to a cycloaddition or ene product. For example, a clockwise rotation of the top ring in rac-8 intermediate $\sim 60$ degrees about C1-C4' and radical recombination at $\mathrm{C} 2$ and C3' generate anti-3. A rotation of the same ring $\sim 120$ degrees and C4-C3' radical recombination yield endo-2. A further clockwise rotation ( 150 degrees) bringing C5 and C1' in proximity could allow H-transfer from C5 to C1', leading to the ene product threo-4. Similar analysis for meso-8 shows that exo-2, syn-3 and erythro-4 can be formed from it, though erythro-4 was not observed experimentally.

Concerted [4+2] cycloadditions vs [6+4]-ene reactions. Figure 5 shows the potential energy surface of various dimerization pathways of 1,3-cyclohexadiene at 1 atm, calculated at the $\omega$ B97XD/def2-TZVP level of theory. Table 1 reports the computed reaction barriers at $\omega$ B97XD, multireference perturbation NEVPT2, and coupled-cluster $\operatorname{CCSD}(\mathrm{T})$ levels. 


\section{Concerted pathways}

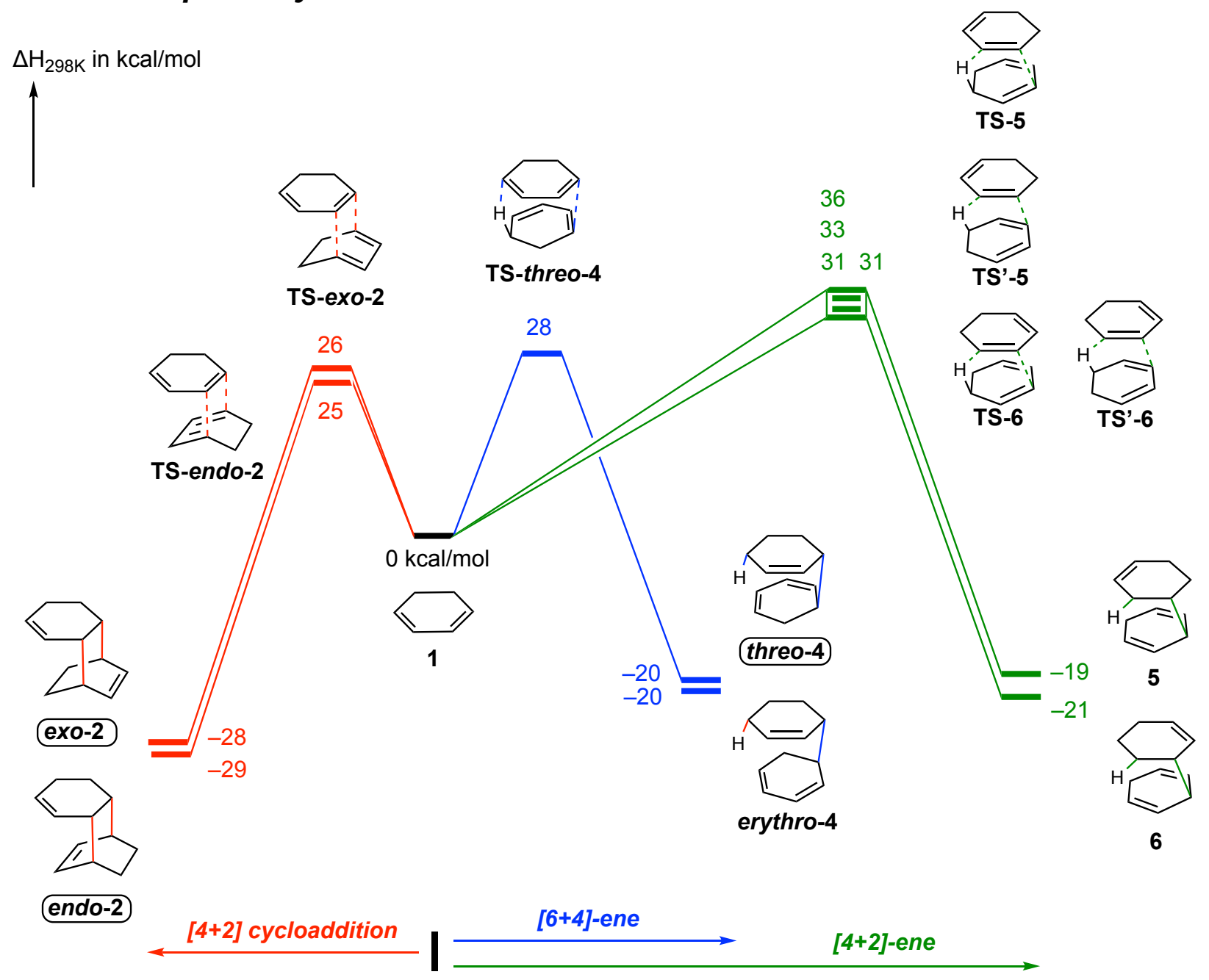

Figure $5.1 \mathrm{~atm}$ enthalpic profiles of concerted pathways calculated at the $\omega$ B97XD/def2TZVP level of theory. Experimentally-observed dimers are indicated by rounded-corner boxes around the labels. 3D drawings of the transition states are given in the Supporting Information.

At the $\omega$ B97XD/def2-TZVP level of theory, the endo [4+2] cycloaddition pathway going through TS-endo-2 is calculated to have the lowest enthalpic barrier of $25 \mathrm{kcal} / \mathrm{mol}$. The barrier of the exo pathway is $1 \mathrm{kcal} / \mathrm{mol}$ higher, likely due to the absence of secondary orbital interactions ${ }^{64}$ in TS-exo-2. $\omega$ B97XD/def2-TZVP calculations also show that the activation enthalpy of the threo [6+4]-ene reaction going through TS-threo-4 is $3 \mathrm{kcal} / \mathrm{mol}$ higher than that of the endo [4+2] cycloaddition. However, CCSD(T)/def2-TZVP single- 
point calculations give opposite results that the threo [6+4]-ene pathway is the most favored pathway, with TS-threo-4 being $1 \mathrm{kcal} / \mathrm{mol}$ lower than TS-endo-2. The CCSD(T) computed activation enthalpies agree excellently with the experimental activation energies (Table 1).

Table 1. Calculated activation enthalpies $\Delta H_{298 K}^{\ddagger}$ and Gibbs activation energy $\Delta G_{298 K}^{\ddagger}$ (in parenthesis), and experimental activation energies $E_{a}$ and pre-exponential factor $\lg A$ of the Arrhenius Equation for the five dimerization mechanisms of cyclohexadiene. Zero-point vibrational, thermal, and entropic corrections are computed at the $\omega$ B97XD/def2-TZVP level. For the stepwise TS structures, the arrow " $\rightarrow$ " points from spin-unprojected to spinprojected energies. All energies are in $\mathrm{kcal} / \mathrm{mol}$.

\begin{tabular}{|c|c|c|c|c|c|c|}
\hline \multirow[t]{2}{*}{ Mechanism } & \multirow[t]{2}{*}{ TS structure } & \multicolumn{3}{|c|}{ Calc. $\Delta H_{298 K}^{\ddagger}\left(\Delta G_{298 K}^{\ddagger}\right)$ in kcal $/ \mathrm{mol}$} & \multirow{2}{*}{$\begin{array}{l}\text { Expt. } E_{a} \text { in } \\
\mathrm{kcal} / \mathrm{mol}\end{array}$} & \multirow[t]{2}{*}{ Expt. $\lg A$} \\
\hline & & $\omega \mathrm{B} 97 \mathrm{XD}^{\mathrm{a}}$ & NEVPT2 $^{b}$ & $\operatorname{CCSD}(\mathrm{T})^{c}$ & & \\
\hline $\begin{array}{l}\text { Concerted } \\
{[4+2]}\end{array}$ & TS-endo-2 & $25(38)$ & $18(31)$ & $22(34)$ & 22.6 & 5.46 \\
\hline $\begin{array}{l}\text { Concerted } \\
{[4+2]}\end{array}$ & TS-exo-2 & $26(40)$ & $20(34)$ & $24(37)$ & n.a. & n.a. \\
\hline $\begin{array}{l}\text { Concerted } \\
{[6+4] \text {-ene }}\end{array}$ & TS-threo-4 & $28(42)$ & $18(33)$ & $21(35)$ & 21.5 & 3.87 \\
\hline Stepwise & TS-meso & $24(36) \rightarrow 18(30)^{d}$ & $19(31)$ & & $24.2 / 24.3$ & $5.51 / 5.10$ \\
\hline Stepwise & TS-rac & $28(40) \rightarrow 21(34)^{\mathrm{e}}$ & $24(37)$ & & 25.3 & 5.52 \\
\hline
\end{tabular}

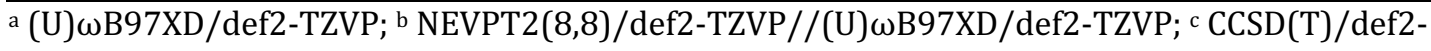

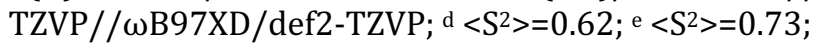

Interestingly, although both experiments and calculations show that the concerted [6+4]-ene reaction (via TS-threo-4) has the lowest activation energy/enthalpy, the corresponding [6+4]-ene adduct threo-4 is only a minor product, comprising $8 \%$ of the observed dimers. Importantly, the product ratio does not change over the reaction course and all dimers are verified to be kinetically persistent, i.e., no interconversion among the dimers. ${ }^{26}$ Therefore, the low product ratio of threo-4 must mean that the [6+4]-ene reaction is relatively slow. Since the rate of a reaction is determined by $\Delta G^{\ddagger}$, not just by $\Delta H^{\ddagger}$ or $E_{a}$, the two observations-the lowest activation energy/enthalpy and a relatively slow 
reaction rate-about the [6+4]-ene reaction can be reconciled by realizing that this reaction has a significantly smaller activation entropy, compared with other reactions. Calculations show that the entropy of TS-threo-4 is $5 \mathrm{~J} / \mathrm{mol} \cdot \mathrm{K}$ smaller than TS-endo-2; this entropic difference corresponds to a difference of $1.5 \mathrm{kcal} / \mathrm{mol}$ in $\Delta G_{298 K}^{\ddagger}$, in favor of TSendo-2. Inclusion of the entropic contribution to the Gibbs activation energy reverses the kinetic preference of [6+4]-ene reaction and [4+2] cycloaddition. The smaller entropy of TS-threo-4 indicates higher structural order, a feature that also relates to the large negative activation volume of the [6+4]-ene reaction. We will discuss the compact geometry of TS-threo-4 in a later section. The difference in activation entropy is also reflected by the pre-exponential factor of the Arrhenius equations for these reactions, according to the thermodynamic formulation of the transition state theory.4,19,20,21,22 Consistent with the computed smaller entropy of TS-threo-4, the [6+4]-ene reaction was experimentally measured to have a significantly smaller pre-exponential factor of the Arrhenius equation $(\lg A=3.87)$, compared with other reactions $(\lg A>5)$.

The [4+2] cycloadducts are computed to be the most thermodynamically stable ones among the 1,3-cyclohexadiene dimers considered. The [6+4]-ene adduct threo-4 is about $10 \mathrm{kcal} / \mathrm{mol}$ less stable, but was observed to be kinetically persistent at $70{ }^{\circ} \mathrm{C}$ up to $3 \mathrm{kbar}$ as well as at $110^{\circ} \mathrm{C}$ and $1 \mathrm{~atm} .{ }^{26}$ At elevated temperature $\left(161^{\circ} \mathrm{C}\right)$, threo-4 does slowly disproportionate into benzene and cyclohexene; a retro-[4+2]-ene mechanism was proposed for this disproportionation. ${ }^{26}$ Our $\operatorname{CCSD}(\mathrm{T})$ calculations (in the supporting information) show that the TS structure of this disproportionation has a high enthalpy of $38 \mathrm{kcal} / \mathrm{mol}$, consistent with the observed very slow disproportionation of threo-4 only at elevated temperatures.

The TS structure of a second concerted [6+4]-ene pathway, TS-erytho-4, was not located on the PES. Attempts by scanning the $\mathrm{C} 1-\mathrm{C1}^{\prime}$ and/or $\mathrm{C} 5-\mathrm{H} / \mathrm{C} 4$ '- $\mathrm{H}$ bonds (see Figure 3 or Figure 6 for the labeling) and using the highest energy structure in the scan as the initial structure for TS optimization lead to either the [4+2] cycloaddition TS structure TSexo-2 or the [4+2]-ene TS structure TS-6, both with lower energies than the initial structure. Figure 6 illustrates the geometric similarity of the three structures; TS-erytho-4 and TS-exo-2 share the same forming C1-C1' bond while TS-erytho-4 and TS-6 share the 
same C5-H...C4' H-transfer moiety. One might have even anticipated formation of an ambimodal transition state leading to several of these products. $65,66,67,68,69,70,71$ The existence of the two lower energy TS structures near TS-erytho-4 on the PES likely makes TS-erytho-4 no longer a stationary point. These calculations are consistent with the [6+4]ene adduct erytho-4 not being observed experimentally.
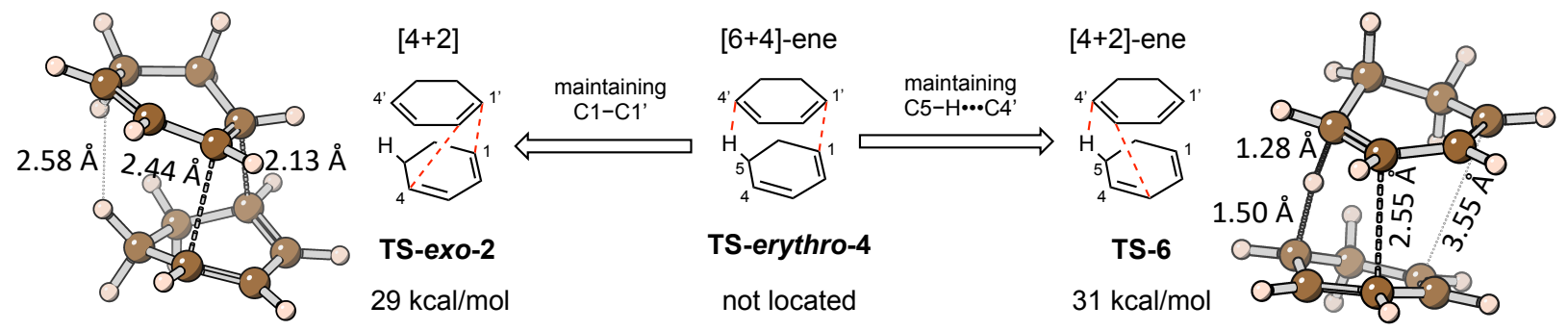

Figure 6. The structural relationship among three similar TS structures. $\omega$ B97XD/def2-TZVP enthalpies $\left(\Delta H_{298 K}\right)$ of the optimized structures are shown.

Concerted [4+2]-ene pathways. As shown in Figure 5, the computed enthalpic barriers of [4+2]-ene reactions are $2-11 \mathrm{kcal} / \mathrm{mol}$ higher than those of the [4+2] cycloaddition and [6+4]-ene pathways. A plausible reason for the higher energies of the $[4+2]$-ene TS structures is that the conjugation between the dienes in the bottom ring (Figure 5) is lost in the [4+2]-ene TS structures whereas it is remained in the TS structures of the [4+2]-cycloadditions and [6+4]-ene reaction. These $>30 \mathrm{kcal} / \mathrm{mol}$ large barriers of the [4+2]-ene reactions are consistent with the fact that [4+2]-ene products are not observed experimentally.

Stepwise pathways. Figure 7 shows the 1 atm enthalpic reaction profiles of the stepwise pathways. The initial $\mathrm{C}-\mathrm{C}$ bond formation between two molecules of $\mathbf{1}$, via the rac addition (the left branch) or the meso addition (the right branch), leads to a diradical intermediate rac-8 or meso-8. TS-meso is computed to be $4 \mathrm{kcal} / \mathrm{mol}$ lower in enthalpy than TS-rac; rac-8 and meso-8 are computed to be similar in enthalpy. rac-8 and $\mathbf{m e s o - 8}$ have anti conformations around the forming $\mathrm{C}-\mathrm{C}$ bonds. The search for stepwise TS structures in gauche conformations lead to concerted TS structures. For example, the two 
gauche conformations of TS-rac evolved into TS-endo-2 and TS-threo-4 during TS optimization. It seems that only the anti conformations of the stepwise TS structures are stationary points on the PES at the $\omega$ B97XD/def2-TZVP level of theory. In the second step, two TS structures starting from rac-8 (or meso-8) were located, affording [2+2]cycloadduct anti-3 (or syn-3) and [4+2]-cycloadduct endo-2 (or exo-2). The [4+2] cycloadducts are computed to be about $8-11 \mathrm{kcal} / \mathrm{mol}$ more stable than the [2+2] cycloadducts.

\section{Stepwise pathways}

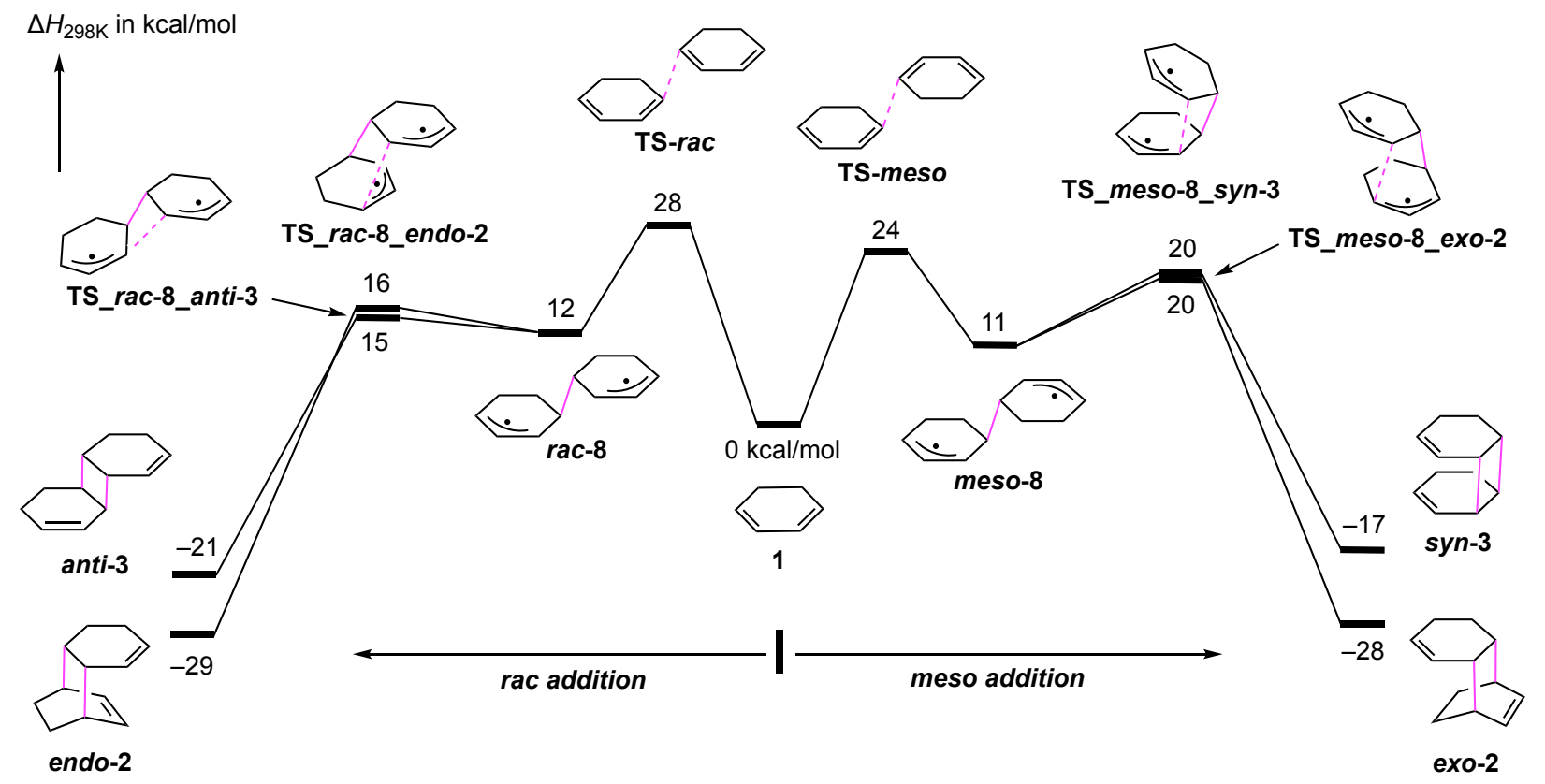

Figure 7. $1 \mathrm{~atm}$ enthalpic reaction profiles of stepwise pathways of 1,3-cyclohexadiene dimerization, calculated at the $\omega \mathrm{B} 97 \mathrm{XD} / \mathrm{def2}$-TZVP level of theory.

Formations of $\boldsymbol{e x o - 2}$ and $\boldsymbol{s y n}-\mathbf{3}$ share the same stepwise meso addition mechanism. Their respective TSs (TS_meso-8_exo-2 and TS_meso-8_syn-3) for the second bond formation from the same intermediate meso-8 were computed to have the same enthalpy of $20 \mathrm{kcal} / \mathrm{mol}$. From the anti conformation of meso-8, more rotation about the first 
formed C $-\mathrm{C}$ bond ( $\sim 180$ degrees, see Figure 3$)$ is necessary in order to reach a geometry for the second bond to form to give syn-3. This might be the reason that syn-3 has a lower product ratio than exo-2 in experiment.

Many structures in the stepwise pathways are open-shell singlet states with significant multi-reference character. Single-reference DFT calculations using the brokensymmetry approach, which is what we used to compute the energies of, for example, TS_meso and TS_rac (Table 1), introduce spin contamination from the triplet to the openshell singlet wave functions, as indicated by the $<\mathrm{S}^{2}>$ values from broken-symmetry DFT calculations, for instance $\left\langle\mathrm{S}^{2}>=0.62\right.$ for TS-meso. The spin-contamination can be removed by the Yamaguchi and Houk spin-projection procedure..$^{53}$

However, either the spin-contaminated or the spin-projected energies shows that the stepwise TS-meso is lower in energy than the concerted TS-endo-2, in contradiction to the experimental activation energies $E_{a}$ in Table 1. In order to compare the computed barriers of the stepwise pathways with the concerted pathways at a consistent level of theory, we resorted to NEVPT2 calculations. These NEVPT2 calculations are based on $\operatorname{CASSCF}(8,8)$ wavefunctions and, in addition to the fully-accounted dynamic electron correlation within the active space, the dynamic electron correlation outside of the active space is recovered through the $2^{\text {nd }}$ order perturbation theory. As shown in Table 1 , TSmeso was computed to be $1 \mathrm{kcal} / \mathrm{mol}$ higher in enthalpy than TS-endo-2 at the

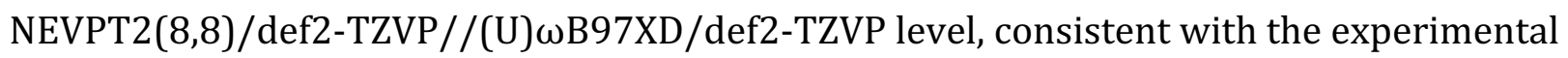
$E_{a}$. However, the 3-5 kcal/mol difference between TS-meso and TS-rac at $\omega$ B97XD and NEVPT2 levels is larger than the experimental $E_{a}$, though this large difference is consistent with previous calculations. ${ }^{28}$

The [4+2] cycloadducts endo-2 and exo-2 may be formed by either a concerted or stepwise mechanism as shown in Figure 3. The NEVPT2 calculations in Table 1 suggest that these two [4+2]-cycloadducts are formed by different mechanisms. For endo-2, the

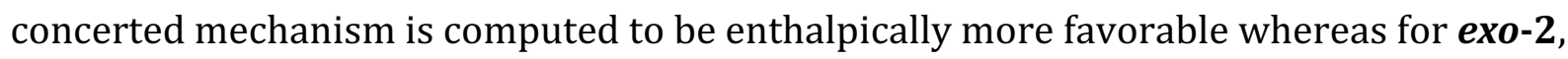
the stepwise mechanism is more favorable. 
Although NEVPT2 calculations distinguishes the concerted and stepwise pathways reasonably well, they underestimate the enthalpic barriers of most pathways by about 4 $\mathrm{kcal} / \mathrm{mol}$, when compared against the experimental activation energy $E_{a}$ (Table 1). We recommend referring to $\operatorname{CSSD}(\mathrm{T})$ barriers for the comparison with experimental $E_{a}$, and to the NEVPT2 barriers for comparing relative barrier heights, especially between the concerted and stepwise pathways. Irrespective of the level of theory, the concerted and stepwise pathways were computed to have enthalpic barriers within $5 \mathrm{kcal} / \mathrm{mol}$. The small enthalpic difference between these TS structures are consistent with the experiment, confirming the competitive nature of the concerted and stepwise mechanisms in this reaction.

We summarize our calculations and discussions on the $1 \mathrm{~atm}$ PES of the thermal dimerization of cyclohexadiene as follows:

- For endo-2, the concerted [4+2] cycloaddition is computed to be more favorable than the stepwise mechanism at both the $\omega$ B97XD (spin-projected) and NEVPT2 $(8,8)$ levels. The CCSD(T) computed $\Delta H_{298 K}^{\ddagger}$ for the concerted [4+2] cycloaddition gives the best agreement with the experimental $E_{a}$.

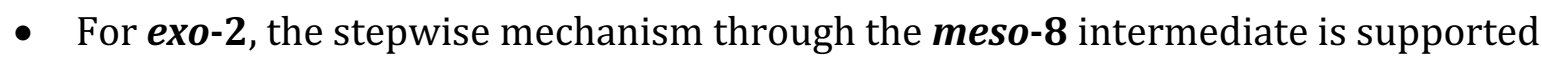
against the concerted $[4+2]$ cycloaddition by both the $\omega$ B97XD and NEVPT2 $(8,8)$ calculations.

- For threo-4, the concerted [6+4]-ene mechanism has the lowest $\Delta H_{298 K}^{\ddagger}$ among all the reactions considered, as shown by the NEVPT2 and CCSD(T) calculations. The $\operatorname{CCSD}(\mathrm{T}) \Delta H_{298 K}^{\ddagger}$ for this reaction is in excellent agreement with the experimental $E_{a}$.

- For syn-3, the thermally allowed stepwise mechanism through the meso-8 intermediate is favored over the concerted mechanism by both the $\omega \mathrm{B} 97 \mathrm{XD}$ (spinprojected) and NEVPT2 $(8,8)$ calculations; however, both methods give an underestimation $(5-6 \mathrm{kcal} / \mathrm{mol})$ of the experimental activation energy. 
- For anti-3, similar as syn-3, only the stepwise mechanism through the rac-8 intermediate is thermally allowed. Only the NEVPT2 $(8,8)$ calculations give a reasonable estimation of the experimental activation energy.

- The above calculations are in good agreement with the previous calculations by Ess et $a .^{28}$ In addition, we explored the plausible [4+2]-ene mechanisms that were not considered before. The large computed barriers (> $31 \mathrm{kcal} / \mathrm{mol}$ at $\omega \mathrm{B} 97 \mathrm{XD} / \mathrm{def} 2-$ TZVP level of theory) are consistent with the fact that the corresponding [4+2]-ene products 5 and $\mathbf{6}$ were not observed experimentally.

\section{Reaction profiles under pressure}

Figure 8 shows the computed profiles of the effective energy $\Delta G_{t o t}$ (see eq. 1 ) and cavity volume (the volume of the vdW cavity hosting the molecule in the continuum; see Computational Methodology for details) profiles of four different types of dimerization of 1,3-cyclohexadiene-the concerted [4+2]-cycloaddition, concerted [6+4]-ene reaction, concerted [4+2]-ene reaction, and the first step of a stepwise addition. For each type of reaction, one stereo version is shown and the others are given in the SI. For example, only the endo [4+2] cycloaddition is shown in Figure 8 and the exo cycloaddition is given in the SI. The profiles at $0 \mathrm{GPa}$ are from the intrinsic reaction coordinate calculations; the highpressure profiles are computed using the XP-PCM method based on the 0 GPa structures.

A first look shows that the four types of reaction have very different profiles, for example, in the location of the TS structure along the reaction coordinate and in the shape of the $\Delta G_{t o t}$ and volume profiles. They do share a common feature-the $\Delta G_{\text {tot }}$ profile decreases as the pressure increases, which is consistent with the expected negative activation volumes of these dimerizations.

Concerted [4+2] cycloaddition. The concerted [4+2] cycloaddition has a fairly late TS at about 2/3 of the entire reaction course. The reaction profile is similar to other [4+2] cycloadditions previously studied using the same XP-PCM method.17,72,73 In the current 
endo-cycloaddition, the first 1/4 of the PES is rather flat, where two isolated 1,3cyclohexadiene molecules reorient themselves in a proper geometry for the cycloaddition and begin to approach one another; the latter motion would lead to a decrease of the separation between the molecules. At high pressure, a minimum of a vdW complex develops prior to the TS, as noted previously by us ${ }^{17,72}$ and by Loco et. al. ${ }^{50}$ The minimum is very shallow at low pressures (may be difficult to see in the figure, but it is there) but becomes apparent at $5.7 \mathrm{GPa}$. This minimum also shifts towards the TS as the pressure increases. The emergence of a pre-TS minimum and the shift of it towards the TS could be explained by the pressure-enhanced vdW complex formation. The higher the pressure, the shorter the vdW separation between the molecules, and the smaller the volume of such complex. The favorable $\mathrm{pV}$ term of the enthalpy leads to the appearance of a minimum for the complex. This phenomenon of a pre-TS minimum seems to be common in bimolecular reactions; the other three reactions in Figure 8 also exhibits such a feature, especially in the concerted [6+4]-ene reaction, where deep pre-TS minima at high pressures are suggested by the calculations. 

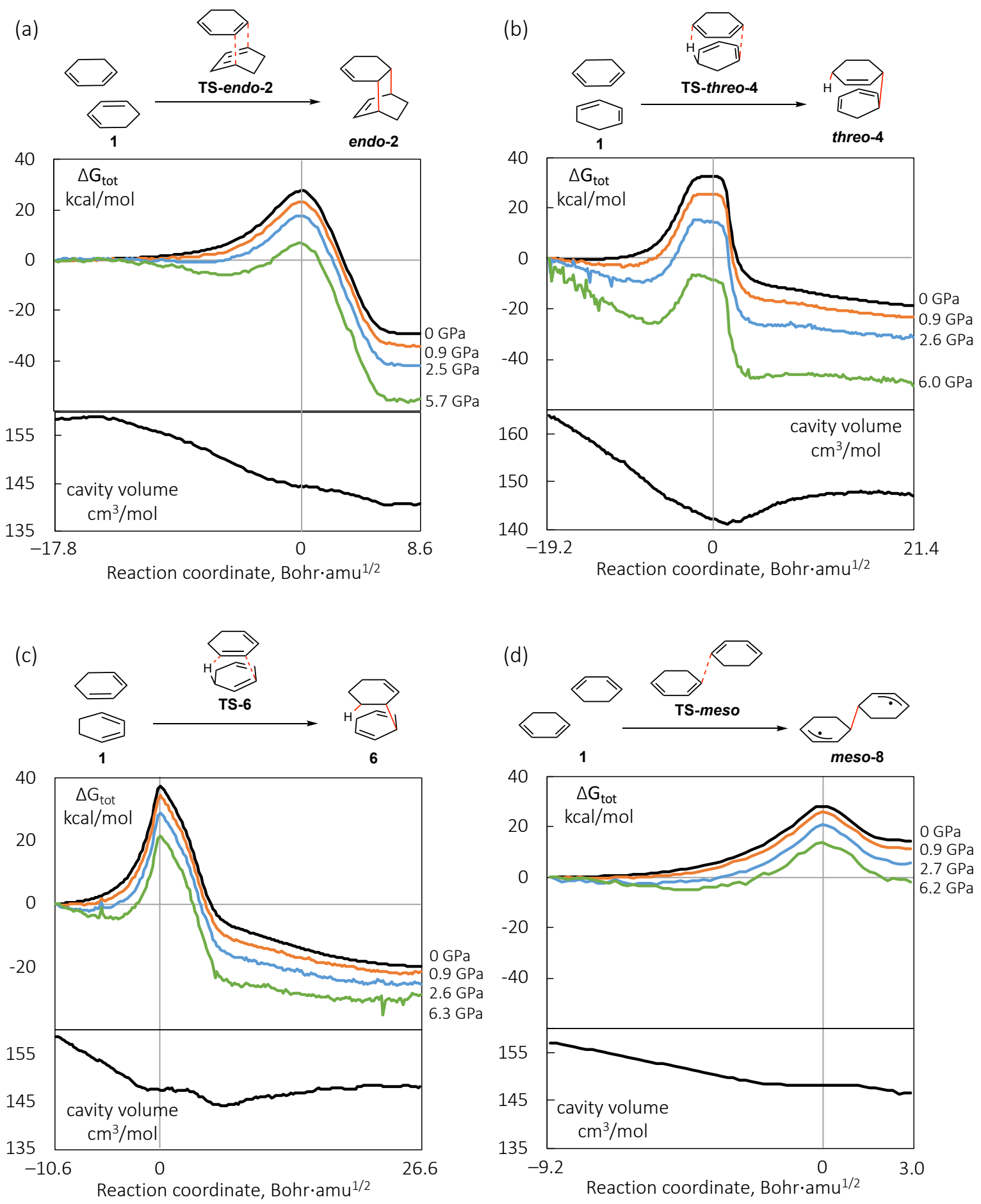

Figure 8. Effective reaction profiles $\left(\Delta G_{\text {tot }}\right.$ in eq. 1$)$ at different pressures and cavity volume profiles (vdW cavity with a scaling factor of 1.2 of the Bondi radii) of concerted [4+2] cycloaddition, concerted [6+4]-ene reaction, and the first step of the meso stepwise addition of cyclohexadiene dimerization, calculated by the XP-PCM method at the $\omega$ B97XD/def2TZVP level. 
The cavity volume profile of the endo [4+2] cycloaddition also shows a flat region at the beginning of the reaction; this is where the two molecules reorient themselves with a rotation before approaching each other. Afterwards, the cavity volume, as defined in the PCM model, decreases monotonically during the course of bond formation and does not change much when the bond formation is complete at the end of the profile. A nice correspondence between the cavity volume profile and the $\Delta G_{\text {tot }}$ profile is evident-at the beginning and end of the reaction, the volume and $\Delta G_{t o t}$ profiles are both flat, whereas during the bond formation, the decreasing volume is matched by the enthalpy decrease at high pressures.

Concerted [6+4]-ene reaction. In the threo type [6+4]-ene reaction (Figure 8b), the concurrent $\mathrm{C}-\mathrm{C}$ bond formation and $\mathrm{H}$-transfer at the 1,4 positions of one ring and 1,5 positions of the other results in a substantial overlap of the two rings in the TS, more than that in the [4+2] cycloaddition TS. A top view (Figure 9) of this TS structure, TS-threo-4, shows that the two rings are almost coaxial and the $\mathrm{CH} / \mathrm{CH}_{2}$ groups are in a staggered conformation. On the contrary, the [4+2] cycloaddition TS has less overlap between the two rings as shown in the top view. The greater overlap of the rings in TS-threo-4 leads to a more compact structure and a smaller volume of the structure, compared with TS-endo-2. The vdW cavity of TS-threo-4 is $142 \mathrm{~cm}^{3} / \mathrm{mol}$, compared with $145 \mathrm{~cm}^{3} / \mathrm{mol}$ for TS-endo-2. Note that $1 \mathrm{~cm}^{3} / \mathrm{mol}$ difference in volume corresponds to $0.24 \mathrm{kcal} / \mathrm{mol}$ difference in enthalpy at $1 \mathrm{GPa}$. The greater overlap of the rings and the highly-ordered staggered conformation in TS-threo-4 is correlated with the small pre-exponential factor (i.e., small entropy of the TS) in the Arrhenius equation for the threo [6+4]-ene reaction (as discussed in the $1 \mathrm{~atm}$ PES section), compared with other reactions. 
Side view

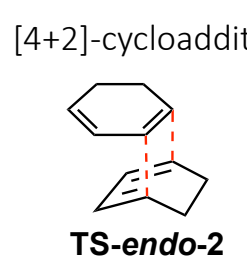

[6+4]-ene

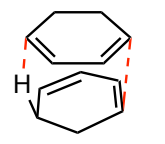

TS-threo-4
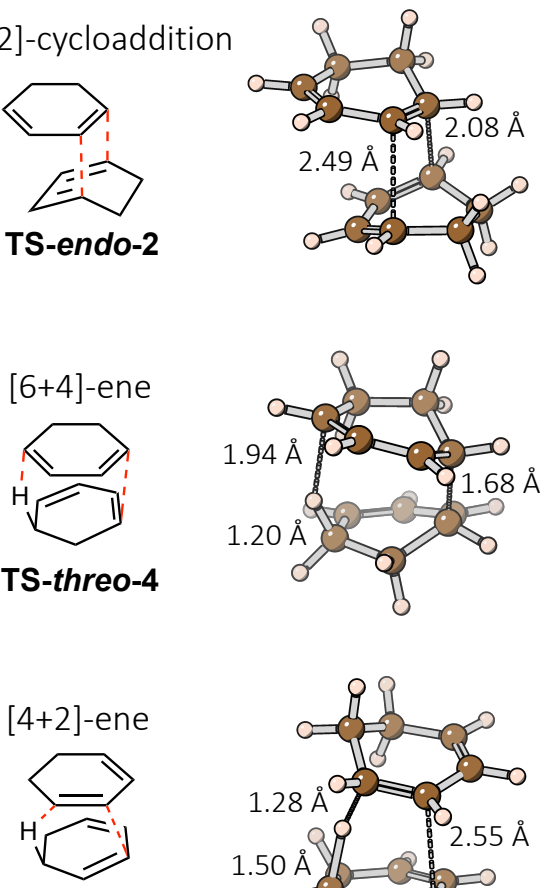

TS-6
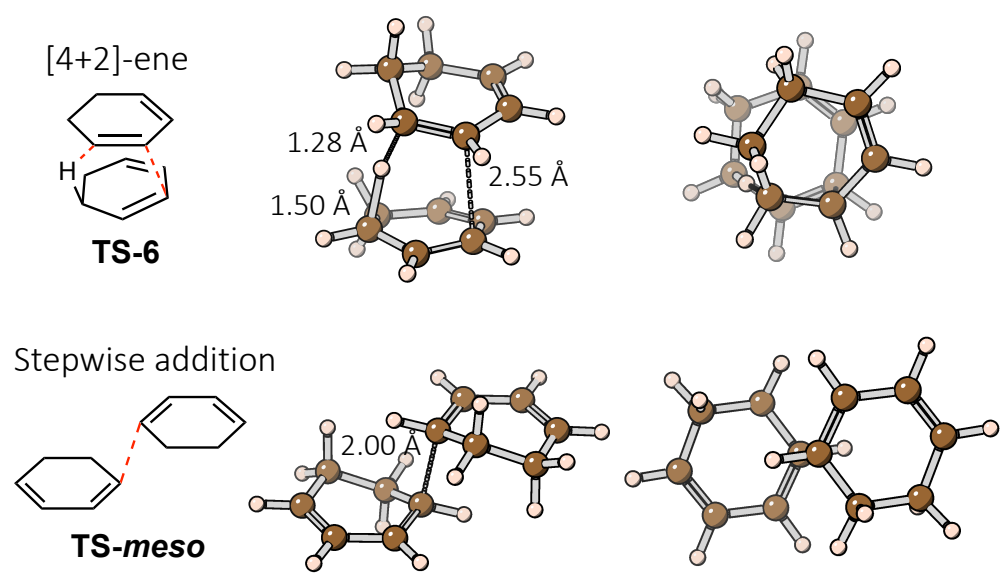

Figure 9. Two views of the $\omega$ B97XD/def2-TZVP optimized gas-phase TS structures with key bond distances shown.

The $0 \mathrm{GPa}$ PES of the threo [6+4]-ene reaction has a rather flat region near the TS (Figure $8 \mathrm{~b}$ ), during which the $\mathrm{C} \cdots \mathrm{C}$ and $\mathrm{C} \cdots \mathrm{H}$ distances of the forming bonds (indicated by red dashes in TS-threo-4) change from $1.9 \AA$ and $2.3 \AA$ to $1.7 \AA$ and $1.8 \AA$, respectively. After the flat region of the PES, the H-transfer completes in a few steps with a fast and substantial energy decrease. Then, a long and slow conformational change takes place in the last $1 / 3$ of the reaction process. This conformation change mainly features the separation of the $\mathrm{C}$, from which the $\mathrm{H}$ is transferred, from the newly formed $\mathrm{C}-\mathrm{H}$ bond. Ignoring the last $1 / 3$ of the profile of conformational change, this reaction also has a very 
late TS. The cavity volume of the system decreases from the beginning of the reaction to about the point at which the H-transfer completes (i.e., a bit pass the TS). Then the cavity increases, as the ene-adduct opens up to adapts to its optimal conformation at $0 \mathrm{GPa}$.

At high pressures, a pre-TS vdW complex minimum develops, similar to the case of the [4+2] cycloaddition. But this minimum in the [6+4]-ene reaction is much deeper compared with that in the [4+2] cycloaddition. A most likely reason is that the separated reactant molecules occupy a larger volume $\left(164 \mathrm{~cm}^{3} / \mathrm{mol}\right)$ than in the [4+2] cycloaddition $\left(158 \mathrm{~cm}^{3} / \mathrm{mol}\right)$, so that a greater volume reduction is possible in forming the vdW complex. The flat TS region of the PES at $0 \mathrm{GPa}$ becomes tilted with the post-TS region lowering in enthalpy at high pressures, due to the monotonical volume decrease in this region. A clear shift of the TS towards the reactant is seen at high-pressures. The shift of TS at high pressure is consistent with the Hammond postulate ${ }^{74}$ that the TS shifts towards the reactant when the reaction becomes more exothermic. However, note that the TS shift is apparent only in the [6+4]-ene reaction, not so in the other three reactions in Figure 8, though all these reactions become more exothermic (or less endothermic) at high pressures. One distinct feature of the [6+4]-ene reaction is that the cavity volume decreases in the TS region, while in the other three reactions, the cavity volume stays almost constant in the TS region. Therefore, the effect of pressure is larger on the PES in the TS region in the [6+4]-ene reaction, leading to an apparent shift of the TS towards the reactant. The shift of TS along reaction coordinate under pressure has been noted previousely. $17,40,48,49$

Concerted [4+2]-ene reaction. In contrast to the flat TS region in the [6+4]-ene reaction, the [4+2]-ene reaction has a sharp maximum in the TS region (Figure 8c). We inspected the TS structures in the two reactions to understand the reason. In the [6+4]-ene TS-threo-4, the C-C bond is almost fully formed at a distance of 1.68 Å while the $\mathrm{H}$ is barely transferred with distances of 1.20 and $1.90 \AA$ for the breaking and forming $\mathrm{C}-\mathrm{H}$ bonds (Figure 9). On the contrary, in the [4+2]-ene TS-6, the H-transfer is substantial with 1.50 and $1.28 \AA$ for the two $\mathrm{C}-\mathrm{H}$ bonds but the $\mathrm{C}-\mathrm{C}$ bond to be formed is at a large distance of $2.55 \AA$ A. The shapes of PESs near the TSs and the structural features of the TSs in these two ene reactions suggest that the $\mathrm{H}$-transfer is associated with a much larger energy change than the $\mathrm{C}-\mathrm{C}$ bond formation. When the TS structure of the ene reaction is dominated by $\mathrm{C}-$ 
$\mathrm{C}$ bond formation, the PES near the TS is flat, as in the [6+4]-ene reaction; when the TS structure is governed by H-transfer, the PES near the TS is sharp, as in the [4+2]-ene reaction.

The [4+2]-ene reaction also has a long course of conformational change in the second half of the profile. Again, this is due to the opening up of the molecule after the $\mathrm{H}$ transfer. Correspondingly, the volume increases during the course of this conformational change (Figure 8c). Interestingly, the volume profile has a flat region near the TS; the reason is two-fold. In the region before the TS, where H-transfer starts to take place, the $\mathrm{C} \cdots \mathrm{C}$ distance in the $\mathrm{C}-\mathrm{H} \cdots \mathrm{C}$ moiety stays almost constant (at about $2.8 \AA$ ), so does the distance of the $\mathrm{C}-\mathrm{C}$ bond to be formed (at about 2.6 $\mathrm{A}$ ). During this course, there is little movement of the two molecules against each other, which leads to little volume change. In the region after the TS, the $\mathrm{C}-\mathrm{C}$ bond starts to form, which reduces the separation between the two rings and should lead to volume reduction. However, this effect is counterbalanced by the opening-up of the $\mathrm{C} \cdots \mathrm{H}-\mathrm{C}$ moiety after $\mathrm{H}$-transfer that results in a volume increase. The overall effect is that the volume stays constant in quite a region after the TS. Afterwards, the $\mathrm{C}-\mathrm{C}$ bond formation dominates for a short duration with a decrease of the cavity volume, before the final conformational change takes over and leads to a slow volume increase.

The cavity volume of the [4+2]-ene TS-6 is $147 \mathrm{~cm}^{3} / \mathrm{mol}$, larger than those of the TS-endo-2 in the [4+2]-cycloaddition $\left(145 \mathrm{~cm}^{3} / \mathrm{mol}\right)$ and TS-threo-4 in the [6+4]-ene reaction $\left(142 \mathrm{~cm}^{3} / \mathrm{mol}\right)$. Consequently, the lowering of the enthalpy at high pressure is much less in the [4+2]-ene TS than in the other two reactions. As discussed above, because the cavity volume does not change in the TS region in the [4+2]-ene reaction, one would not expect a shift the TS location under pressure (Figure 8c).

Stepwise addition. Moving on to the stepwise mechanism, Figure $8 \mathrm{~d}$ shows the reaction profiles of the meso addition. Compared with the above-discussed concerted reactions, this meso addition has a rather short course, especially in the post-TS region. The C-C distance of the formed bond changes from $2.00 \AA ̊$ in TS-meso to $1.57 \AA$ in the adduct meso-8. The cavity volume of the system decreases in the meso-addition, but with a much 
less volume reduction compared with the above concerted reactions where two bonds are formed. Consequently, pressure provides much less reduction of the reaction barrier for the meso addition. The meso-adduct meso-8 is calculated to be higher in enthalpy than the reactants at pressures lower than $6.2 \mathrm{GPa}$.

\section{Calculations of activation volumes}

In addition to activation energy, activation volume also provides information about the transition state, arguably, in a more tangible manner. The volume/size of a TS structure is directly related to its geometry. While competing mechanisms of a reaction can sometimes be difficult to distinguish based on activation energies, they may be distinguishable by activation volumes when the competing TS structures have very different geometries. This is especially useful in studying the stepwise vs concerted mechanisms of cycloadditions, given that the stepwise mechanism often has a less negative activation volume..$^{24,25}$

In order to calculate the activation volume of a reaction, the barriers computed by XP-PCM are plotted against the pressure (Figure 10). The $\Delta G_{t o t}^{\ddagger}(p)$ data shows a nice linear relationship. The slope of the fitted line gives the activation volume, according to eq. (2) . Note that the free energy $\Delta G_{\text {tot }}^{\ddagger}(p)$ does not contain zero-point vibrational correction and thermal correction, assuming their negligible contributions to $\Delta V^{\ddagger}$. The components of $\Delta G^{\ddagger}$ are the electronic energy and cavitation energy computed by XP-PCM (eq. 1). Note that for the $\Delta V^{\ddagger}$ calculations, $\Delta G_{t o t}^{\ddagger}$ at different pressures are computed using as the reference isolated reactant molecules, not the vdW complexes (pre-TS minima) in Figure 8. This is to ensure a common reference (isolated reactants) in the $\Delta V^{\ddagger}$ comparison for different mechanisms. 


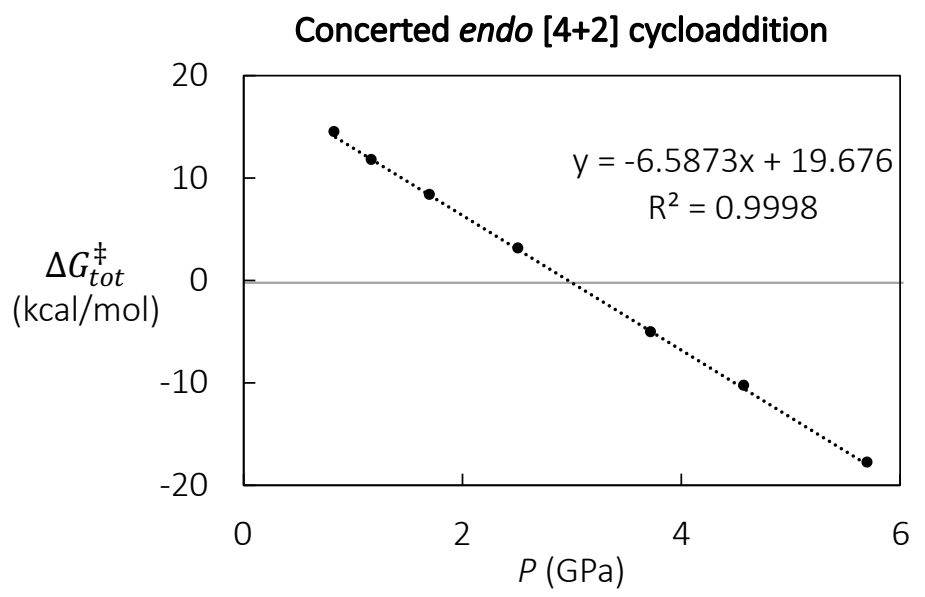

Figure 10. The plot of computed activation energies at different pressures $\Delta G_{\text {tot }}^{\ddagger}(p)$ in the concerted endo [4+2]-cycloaddition, for computing activation volume $\Delta V^{\ddagger}$.

Table 2 shows the computed activation volumes $\Delta V^{\ddagger}$ from linear fitting of the computed $\Delta G_{\text {tot }}^{\ddagger}$ at different pressures, which are compared with experimental values.

The computed $\Delta V^{\ddagger}$ are -27.7 and $-28.2 \mathrm{~cm}^{3} / \mathrm{mol}$ for the concerted endo and exo [4+2] cycloadditions. Less negative $\Delta V^{\ddagger}$ of -20.5 and $-21.4 \mathrm{~cm}^{3} / \mathrm{mol}$ are computed for the stepwise mechanisms that may also deliver the two [4+2] cycloadducts. Comparison with experimental $\Delta V^{\ddagger}$ highly suggests that the endo [4+2] cycloaddition follows a concerted mechanism, but the exo adduct is formed through a stepwise mechanism.

The concerted [6+4]-ene reaction is computed to have the most negative $\Delta V^{\ddagger}$ of $32.1 \mathrm{~cm}^{3} / \mathrm{mol}$, in excellent with the experimental value, thus conforming the concerted mechanism of this reaction. This large negative $\Delta V^{\ddagger}$ for the [6+4]-ene reaction is consistent with the compact geometry of the TS structures, as discussed in the reaction profile under pressure section.

For the [2+2]-cycloadduct syn-3, the computed $\Delta V^{\ddagger}$ of the stepwise mechanism through the meso-8 intermediate is in excellent agreement with the corresponding experimental data. For anti-3. The computed $\Delta V^{\ddagger}$ of the stepwise mechanism through the rac-8 intermediate is in good agreement with the experimental data with a slight 
overestimation. These calculations support the stepwise mechanisms for the [2+2] cycloadditions.

Table 2. Calculated ( $\omega$ B97XD/def2-TZVP) and experimental activation volumes $\Delta V^{\ddagger}$.

\begin{tabular}{lll}
\hline Reaction product & computed $\Delta V^{\ddagger}\left(\mathrm{cm}^{3} / \mathrm{mol}\right)$ & experimental $\Delta V^{\ddagger}\left(\mathrm{cm}^{3} / \mathrm{mol}\right)$ \\
\hline endo-2 [4+2]-cycloadduct & -27.7 (concerted) & -28 \\
& -20.5 (stepwise via TS-rac) & \\
\hline exo-2 [4+2]-cycloadduct & -28.2 (concerted) & -22 \\
& -21.4 (stepwise via TS-meso) \\
\hline threo-4 [6+4]-ene adduct & -32.1 (concerted) & -32 \\
\hline syn-3 [2+2]-cycloadduct & -21.4 (stepwise via TS-meso) & -22 \\
\hline anti-3 [2+2]-cycloadduct & -20.5 (stepwise via TS-rac) & -18 \\
\hline
\end{tabular}

\section{Summary and Conclusions}

This work is part of our continuing efforts to apply a new quantum chemical method, the extreme pressure polarizable continuum model (XP-PCM), to the study of reaction energy profiles under pressure, and to calculate activation volumes. The method has been tutorially reviewed and applied by the present authors and Hoffmann ${ }^{17}$ in 2017 to a set of pericyclic reactions where interesting phenomena on reaction energy profiles under pressure were revealed by calculations, such as the shift of the transition state, changes in the rate-determining step, etc. In addition, a reasonable agreement of the computed activation volumes with the experimental values was discovered. The evolution of the van der Waals volume of the reactive system upon proceeding from the reactants to products, as a chemical relevant diagnostic to analyze the effect of the pressure on the reaction energy profile, was emphasized.

In this work, the XP-PCM computational method is applied to the study of the pressure effects on the competing mechanisms of the thermal dimerization of the 1,3- 
cyclohexadiene, providing, for the first time, accurate calculations of activation volumes, compared with the experimental ones. The activation volumes are of primary relevance for the determination of the competing mechanisms in this reaction.

The thermal dimerization of 1,3-cyclohexadiene has been studied experimentally in solution and at various conditions of temperature and pressure, by Klärner et al. ${ }^{26}$ in 1986 and computationally by Ess et al. ${ }^{28}$ in 2008 in the gas phase (1 atm). In experiment, the observed products were from symmetry allowed [4+2] cycloadditions (endo-2, exo-2), $[6+4]$ ene reaction (threo-4) and symmetry-forbidden [2+2] cycloadditions (syn-3, anti-3). While the thermal [2+2] cycloadditions are usually expected to be formed via a stepwise mechanism, all the other products can be formed through competing, concerted or stepwise mechanisms. Based on a simple interpretation of the experimental activation volume (all negative) and of the activation energy, Klärner et al. heuristically suggested that the dimerizations to endo-2 and threo-4 follow a concerted mechanism, while the reaction affording exo-2 follows a stepwise mechanism that is in common with the mechanism leading to the syn-3 [2+2]-adduct. The 2008 computational study of the dimerization of the 1,3-cyclohexadiene focused on the activation energies; the reaction barriers for the competing concerted and stepwise mechanism were computed to be within a range of $5 \mathrm{kcal} / \mathrm{mol}$, confirming the competitive nature between these mechanisms, but with slight differences from the experiment in what concerns the order of the activation energies.

With respect to the previous computational study by Ess et al, we have first expanded the exploration of the reaction energy profile of various mechanisms of the dimerization of the 1,3-cyclohexadiene in the gas phase, in searching for both a more accurate evaluation of the activation energies and a [4+2]-ene mechanism not considered previously. Then, we have applied the XP-PCM method to study the effect of the pressure on the reaction energy profile and to compute the activation volumes.

The gas-phase transition structure for the various concerted and stepwise mechanisms have been evaluated at the $\omega$ B97XD/def2-TZVP level and the corresponding activation energies computed at the NEVPT2(8,8)/def2-TZVP level, and the CCSD(T)/def2- 
TZVP level for the concerted only mechanisms. The comparison between the NEVPT2 $(8,8)$ activation energies in the concerted and stepwise mechanism and the experimental data gives support that products endo-2 and threo-4 follow a concerted mechanism, while the product exo-2 follow a stepwise mechanism in common with the mechanism leading to the syn-3 adduct, with an almost correct order in agreement with the experimental data. The $\operatorname{CCSD}(\mathrm{T})$ give the most accurate activation energies for the endo [4+2] cycloaddition and the [6+4]-ene reaction. For the [4+2]-ene mechanism not considered in the Ess et al. computational study, we have obtained the highest activation energies, consistently with the fact that the related products ( 5 and $\mathbf{6}$ ) were not observed experimentally.

The effect of the pressure on the energy profile has been evaluated using the XPPCM method along with the gas phase intrinsic reaction path (at $\omega$ B97XD/def2-TZVP level) of the concerted endo-2 [4+2] cycloaddition, the concerted [6+4]-ene and [4+2]-ene reactions and for the first step of the stepwise mechanism leading to the exo-2 and syn-3 adducts. The effect of the pressure on the energy profile has been correlated with the profile of the volume of the van der Waals cavity enclosing the reacting systems. The energy profiles of these four types of reactions share a common feature: the energy profile decreases as the pressure increases, consistent with the expected negative activation volumes of these dimerizations. Furthermore, they are characterized by the emergence of a pre-TS minimum, corresponding to a van der Waals complex, and the shift of it toward the transition state, a feature that can be explained by the smaller volume of such complex with respect to the separated reactant and by the corresponding favorable (i.e. negative) enthalpic $\mathrm{pV}$ term to the energy profile. Except for the concerted [6+4]-ene reaction, all other reactions do not present, in the range of pressure (1-6 GPa) explored, a significant shift of the location of the transition state along the reaction coordinate. The shift at high pressure of the transition state of the concerted [6+4]-ene reaction is consistent with the Hammond postulate that the TS shifts towards the reactant when the reaction is more exothermic. Furthermore, it may be related to the fact that concerted [6+4]-ene reaction features a decrease of the vdW cavity volume of the reactant systems in the TS region while in the other reactions, the vdW cavity volume stays almost constant. 
The activation volumes have been computed as the derivative with respect to the pressure of the activation energy extracted from the energy profiles of the various possible mechanism as a function of the pressure for all the five observed adducts, for which the experimental data are available. The comparison between the computed activation volume for the alternative mechanism and experimental data shows in a very clear way that the products endo-2 and threo-4 are compatible only with a concerted mechanism, while the product exo-2 is compatible only with a stepwise mechanism in common with the syn-3 product. Also the computed activation volume for the stepwise mechanism for the anti-3 product is in good agreement with the experimental data.

As in the case of dimerization of the 1,3-cyclohexadiene the experimental activation volumes (and not the activation energies) have been the primary basis for the heuristic suggestion of the mechanisms in operation for this reaction, we may consider the agreement between the computed and experimental activation volumes a more direct validation of the suggested mechanisms.

\section{Acknowledgement}

We thank Roald Hoffmann and Frank Klärner for helpful discussions. R.C. thanks the support of the framework of the COMP-HUB Initiative of the Department of Chemistry, Life Sciences and Environmental Sustainability of the University of Parma, as part of the 'Departments of Excellence' program of the Italian Ministry for Education, University and Research (MIUR, 2018-2022).

\section{Supporting Information}

- 3D drawings of transition state structures, a more detailed description of the XPPCM method, PES of the disproportionation of threo-4, and supplementary reaction profiles to Figure 8 (PDF). 
- Raw output files of calculations containing optimized geometries and absolute energies (ZIP).

\section{References}

${ }^{1}$ Arrhenius, S. Über Die Dissociationswärme Und Den Einfluss Der Temperatur Auf Den Dissociationsgrad Der Elektrolyte. Zeitschrift Für Physikalische Chemie 1889, 4U (1), 96116. https://doi.org/10.1515/zpch-1889-0408.

${ }^{2}$ Arrhenius, S. Über Die Reaktionsgeschwindigkeit Bei Der Inversion von Rohrzucker Durch Säuren. Zeitschrift Für Physikalische Chemie 1889, 4 (1). https://doi.org/10.1515/zpch1889-0116.

${ }^{3}$ Bell, R. P. The Tunnel Effect in Chemistry. 1980. https://doi.org/10.1007/978-1-48992891-7.

${ }^{4}$ Glasstone, S.; Laidler, K. J.; Eyring, H. The Theory of Rate Processes: The Kinetics of Chemical Reactions, Viscosity, Diffusion and Electrochemical Phenomena; McGraw-Hill Book Company: New York, 1941.

${ }^{5}$ Noble, W. J. L. Kinetics of Reactions in Solutions Under Pressure; Progress in Physical Organic Chemistry; John Wiley \& Sons, Inc., 1967; Vol. 5, pp 207-330.

https://doi.org/10.1002/9780470171844.ch3.

${ }^{6}$ Asano, T.; Noble, W. J. L. Activation and Reaction Volumes in Solution. Chem Rev 1978, 78 (4), 407-489. https://doi.org/10.1021/cr60314a004.

${ }^{7}$ Eldik, R. V.; Asano, T.; Noble, W. J. L. Activation and Reaction Volumes in Solution. 2. Chem Rev 1989, 89 (3), 549-688. https://doi.org/10.1021/cr00093a005.

${ }^{8}$ Drljaca, A.; Hubbard, C. D.; Eldik, R. van; Asano, T.; Basilevsky, M. V.; Noble, W. J. le. Activation and Reaction Volumes in Solution. 3. Chem Rev 1998, 98 (6), 2167-2290. https://doi.org/10.1021/cr970461b. 
${ }^{9}$ Basilevsky, M. V.; Weinberg, N. N.; Zhulin, V. M. Pressure Dependence of Activation and Reaction Volumes. J Chem Soc Faraday Transactions 1 Phys Chem Condens Phases 1985, 81 (4), 875-884. https://doi.org/10.1039/f19858100875.

${ }^{10}$ Basilevskii, M. V.; Ryaboi, V. M.; Weinberg, N. N. Nonequilibrium Effects in Kinetics of Cycloaddition Reactions under High Pressure. J Phys Chem 1991, 95 (14), 5533-5540. https://doi.org/10.1021/j100167a032.

${ }^{11}$ Basilevsky, M. V.; Weinberg, N. N. A Microscopic Theory of Chemical Reactions under High Pressures. Can J Phys 1995, 73 (5-6), 267-272. https://doi.org/10.1139/p95-038.

12 Jenner, G. High Pressure Kinetic Investigations in Organic and Macromolecular Chemistry. Angewandte Chemie International Edition in English 1975, 14 (3), 137-143. https://doi.org/10.1002/anie.197501371.

13 Jenner, G. High Pressure Chemistry, Biochemistry and Materials Science. In High Pressure Kinetic Effects as Mechanistic Probes in Organic Chemistry; Winter, R., Jonas, J., Eds.; NATO ASI Series (Series C: Mathematical and Physical Sciences); Springer: Dordrecht, 1993; Vol. 401, pp 345-366. https://doi.org/10.1007/978-94-011-1699-2_18.

14 Jenner, G. Activation of Organic Reactions. High Pressure vs New Emerging Activation Modes; High Pressure Molecular Science; Springer Netherlands, 1999; pp 313-330. https://doi.org/10.1007/978-94-011-4669-2_18.

15 Wurche, F.; Klärner, F.-G. The Effect of Pressure on Organic Reactions: Basic Principles and Mechanistic Applications; High Pressure Chemistry; Wiley-VCH Verlag GmbH, 2002; pp 41-96. https://doi.org/10.1002/9783527612628.ch02.

${ }^{16}$ Hugelshofer, C.; Magauer, T. High-Pressure Transformations in Natural Product Synthesis. Synthesis 2014, 46 (10), 1279-1296. https://doi.org/10.1055/s-0033-1341073.

${ }^{17}$ Chen, B.; Hoffmann, R.; Cammi, R. The Effect of Pressure on Organic Reactions in Fluids-a New Theoretical Perspective. Angewandte Chemie International Edition in English 2017, 56 (37), 11126-11142. https://doi.org/10.1002/anie.201705427.

${ }_{18}$ Margetic, D. High Pressure Organic Synthesis; De Gruyter: Berlin, Boston, 2019. https://doi.org/10.1515/9783110556841.

${ }^{19}$ Steinfeld, J. I.; Francisco, J. S.; Hase, W. L. Chemical Kinetics and Dynamics; Upper Saddle River, N.J. : Prentice Hall, 1999. (see Eq. 10.54); 
${ }^{20}$ IUPAC. Compendium of Chemical Terminology, 2nd ed. (the "Gold Book"). Compiled by A. D. McNaught and A. Wilkinson. Blackwell Scientific Publications, Oxford (1997). Online version (2019-) created by S. J. Chalk. ISBN 0-9678550-9-8.

https://doi.org/10.1351/goldbook.

${ }^{21}$ Eyring, H.; Lin, S. H.; Lin, S. M. Basic Chemical Kinetics; Wiley: New York, 1980. pp.422426.

${ }^{22}$ Laidler, K. J. Chemical Kinetics, 3rd Edition; Pearson, 1987.

${ }^{23}$ Mozurkewich, M.; Benson, S. W. Negative Activation Energies and Curved Arrhenius Plots. 1. Theory of Reactions over Potential Wells. J Phys Chem 1984, 88 (25), 6429-6435. https://doi.org/10.1021/j150669a073.

${ }^{24}$ Eckert, C. A.; Grieger, R. A. Mechanistic Evidence for the Diels-Alder Reaction from HighPressure Kinetics. J Am Chem Soc 1970, 92 (24), 7149-7153. https://doi.org/10.1021/ja00727a021.

${ }^{25}$ Klaerner, F.-G.; Krawczyk, B.; Ruster, V.; Deiters, U. K. Evidence for Pericyclic and Stepwise Processes in the Cyclodimerization of Chloroprene and 1,3-Butadiene from Pressure Dependence and Stereochemistry. Experimental and Theoretical Volumes of Activation and Reaction. J Am Chem Soc 1994, 116 (17), 7646-7657. https://doi.org/10.1021/ja00096a023.

${ }^{26}$ Klärner, F.; Dogan, B. M. J.; Ermer, O.; Doering, W. von E.; Cohen, M. P. Mechanism of the Thermal 1,3-Cyclohexadiene Dimerization: A Non-Concerted Diels-Alder Reaction Leading to the Exo-[4+2] Adduct and a Novel [6+4]-Ene Reaction. Angewandte Chemie Int Ed Engl 1986, 25 (1), 108-110. https://doi.org/10.1002/anie.198601081.

27 Wodward, R. B.; Hoffmann, R. The Conservation of Orbital Symmetry. Angewandte Chemie International Edition in English 1969, 8 (11), 781-853.

https://doi.org/10.1002/anie.196907811.

${ }^{28}$ Ess, D. H.; Hayden, A. E.; Klärner, F.-G.; Houk, K. N. Transition States for the Dimerization of 1,3-Cyclohexadiene: A DFT, CASPT2, and CBS-QB3 Quantum Mechanical Investigation.J Org Chem 2008, 73 (19), 7586-7592. https://doi.org/10.1021/jo8011804.

${ }^{29}$ Klärner, F.-G.; Wurche, F. The Effect of Pressure on Organic Reactions. Journal für praktische Chemie 2000, 342 (7), 609-636. https://doi.org/10.1002/15213897(200009)342:7<609::aid-prac609>3.3.co;2-q. 
${ }^{30}$ Cammi, R. A New Extension of the Polarizable Continuum Model: Toward a Quantum Chemical Description of Chemical Reactions at Extreme High Pressure. J Comput Chem 2015, 36 (30), 2246-2259. https://doi.org/10.1002/jcc.24206.

${ }^{31}$ Stewart, S. G.; Harfoot, G. J.; McRae, K. J.; Teng, Y.; Yu, L.-J.; Chen, B.; Cammi, R.; Coote, M. L.; Banwell, M. G.; Willis, A. C. High-Pressure-Promoted and Facially Selective Diels-Alder Reactions of Enzymatically Derived Cis-1,2-Dihydrocatechols and Their Acetonide Derivatives: Enantiodivergent Routes to Homochiral and Polyfunctionalized Bicyclo[2.2.2] Octenes. The Journal of Organic Chemistry 2020, 85 (20), 13080-13095. https://doi.org/10.1021/acs.joc.0c01767.

${ }^{32}$ Fukuda, R.; Nakatani, K. Quantum Chemical Study on the High-Pressure Effect for [4 + 4] Retrocycloaddition of Anthracene Cyclophane Photodimer. J Phys Chem C 2019, 123 (7), 4493-4501. https://doi.org/10.1021/acs.jpcc.8b10825.

${ }^{33}$ Pagliai, M.; Cammi, R.; Cardini, G.; Schettino, V. XP-PCM Calculations of High Pressure Structural and Vibrational Properties of P 4 S 3. J Phys Chem 2016, 120 (27), 5136-5144. https://doi.org/10.1021/acs.jpca.6b00590.

${ }^{34}$ Caratelli, C.; Cammi, R.; Chelli, R.; Pagliai, M.; Cardini, G.; Schettino, V. Insights on the Realgar Crystal Under Pressure from XP-PCM and Periodic Model Calculations. J Phys Chem 2017, 121 (46), 8825-8834. https://doi.org/10.1021/acs.jpca.7b08868.

${ }^{35}$ Cammi, R. Linear Chains of Hydrogen Molecules under Pressure: An Extreme-Pressure Continuum Model Study.J Chem Phys 2019, 150 (16), 164122.

https://doi.org/10.1063/1.5075511.

36 Cammi, R.; Cappelli, C.; Mennucci, B.; Tomasi, J. Calculation and Analysis of the Harmonic Vibrational Frequencies in Molecules at Extreme Pressure: Methodology and Diborane as a Test Case. J Chem Phys 2012, 137 (15), 154112. https://doi.org/10.1063/1.4757285.

${ }^{37}$ Pagliai, M.; Cardini, G.; Cammi, R. Vibrational Frequencies of Fullerenes C60 and C70 under Pressure Studied with a Quantum Chemical Model Including Spatial Confinement Effects. J Phys Chem 2014, 118 (27), 5098-5111. https://doi.org/10.1021/jp504173k.

${ }^{38}$ Boccalini, M.; Cammi, R.; Pagliai, M.; Cardini, G.; Schettino, V. Toward an Understanding of the Pressure Effect on the Intramolecular Vibrational Frequencies of Sulfur Hexafluoride. J Phys Chem 2021, 125 (29), 6362-6373. https://doi.org/10.1021/acs.jpca.1c02595.

${ }^{39}$ Fukuda, R.; Ehara, M.; Cammi, R. Modeling Molecular Systems at Extreme Pressure by an Extension of the Polarizable Continuum Model (PCM) Based on the Symmetry-Adapted 
Cluster-Configuration Interaction (SAC-CI) Method: Confined Electronic Excited States of Furan as a Test Case. J Chem Theory Comput 2015, 11 (5), 2063-2076.

https://doi.org/10.1021/ct5011517.

${ }^{40}$ Jha, S. K.; Brown, K.; Todde, G.; Subramanian, G. A Mechanochemical Study of the Effects of Compression on a Diels-Alder Reaction.J Chem Phys 2016, 145 (7), 074307.

https://doi.org/10.1063/1.4960955.

${ }^{41}$ Stauch, T. A Mechanochemical Model for the Simulation of Molecules and Molecular Crystals under Hydrostatic Pressure. J Chem Phys 2020, 153 (13), 134503. https://doi.org/10.1063/5.0024671.

${ }^{42}$ Stauch, T. Quantum Chemical Modeling of Molecules under Pressure. International Journal of Quantum Chemistry 2021, 121 (3), e26208. https://doi.org/10.1002/qua.26208.

${ }^{43}$ Boscoboinik, A.; Olson, D.; Adams, H.; Hopper, N.; Tysoe, W. T. Measuring and Modelling Mechanochemical Reaction Kinetics. Chem Commun 2020, 56 (56), 7730-7733. https://doi.org/10.1039/d0cc02992k.

${ }^{44}$ Scheurer, M.; Dreuw, A.; Epifanovsky, E.; Head-Gordon, M.; Stauch, T. Modeling Molecules under Pressure with Gaussian Potentials. Journal of Chemical Theory and Computation 2021, 17 (1), 583-597. https://doi.org/10.1021/acs.jctc.0c01212.

${ }^{45}$ Deglint, E.; Martens, H.; Edwards, E.; Boon, N.; Dance, P.; Weinberg, N. Molecular Dynamics Calculation of Activation Volumes. Phys Chem Chem Phys 2010, 13 (2), 438-440. https://doi.org/10.1039/c0cp01570a.

${ }^{46}$ Spooner, J.; Wiebe, H.; Boon, N.; Deglint, E.; Edwards, E.; Yanciw, B.; Patton, B.; Thiele, L.; Dance, P.; Weinberg, N. Molecular Dynamics Calculation of Molecular Volumes and Volumes of Activation. Phys Chem Chem Phys 2012, 14 (7), 2264-2277.

https://doi.org/10.1039/c2cp22949h.

${ }^{47}$ Spooner, J.; Yanciw, B.; Wiebe, B.; Weinberg, N. Reaction Profiles and Energy Surfaces of Compressed Species. J Phys Chem 2014, 118 (4), 765-777.

https://doi.org/10.1021/jp410496t.

${ }^{48}$ Spooner, J.; Smith, B.; Weinberg, N. Effect of High Pressure on the Topography of Potential Energy Surfaces. Can J Chem 2016, 94 (12), 1057-1064.

https://doi.org/10.1139/cjc-2016-0295. 
${ }^{49}$ Plotnikov, N. V.; Martinez, T. J. Molecular Origin of Mechanical Sensitivity of the Reaction Rate in Anthracene Cyclophane Isomerization Reveals Structural Motifs for Rational Design of Mechanophores. J Phys Chem C 2016, 120 (32), 17898-17908. https://doi.org/10.1021/acs.jpcc.6b04924.

${ }^{50}$ Loco, D.; Spezia, R.; Cartier, F.; Chataigner, I.; Piquemal, J.-P. Solvation Effects Drive the Selectivity in Diels-Alder Reaction under Hyperbaric Conditions. Chem Commun 2020, 56 (49), 6632-6635. https://doi.org/10.1039/d0cc01938k.

${ }^{51}$ Chai, J.-D.; Head-Gordon, M. Long-range corrected hybrid density functionals with damped atom-atom dispersion corrections. Physical Chemistry Chemical Physics 2008, 10 (44), 6615-6620 DOI: 10.1039/b810189b.

${ }^{52}$ Weigend, F.; Ahlrichs, R. Balanced basis sets of split valence, triple zeta valence and quadruple zeta valence quality for $\mathrm{H}$ to $\mathrm{Rn}$ : Design and assessment of accuracy. Physical Chemistry Chemical Physics 2005, 7 (18), 3297-3305 DOI: 10.1039/b508541a.

${ }^{53}$ Yamaguchi, K.; Takahara, Y.; Fueno, T.; Houk, K. N. Extended Hartree-Fock (EHF) Theory of Chemical Reactions. Theor Chim Acta 1988, 73 (5-6), 337-364.

https://doi.org/10.1007/bf00527740.

${ }^{54}$ Angeli, C.; Cimiraglia, R.; Evangelisti, S.; Leininger, T.; Malrieu, J.-P. J. Chem. Phys., 2001, $114,10252$.

${ }^{55}$ Angeli, C.; Cimiraglia, R.; Malrieu, J.-P. Chem. Phys. Lett., 2001, 350, 297.

${ }^{56}$ Angeli, C.; Cimiraglia, R.; Malrieu, J.-P. J. Chem. Phys., 2002, 117, 9138.

${ }^{57}$ Purvis, G. D., III; Bartlett, R. J. A full coupled-cluster singles and doubles model: The inclusion of disconnected triples. J. Chem. Phys. 1982, 76 (4), 1910-1918 DOI: $10.1063 / 1.443164$.

${ }^{58}$ Pople, J. A.; Head-Gordon, M.; Raghavachari, K. Quadratic configuration interaction. A general technique for determining electron correlation energies. J. Chem. Phys. 1987, 87 (1), 5968-5975 DOI: 10.1063/1.453520.

${ }^{59}$ Frisch, M. J.; Trucks, G. W.; Schlegel, H. B.; Scuseria, G. E.; Robb, M. A.; Cheeseman, J. R.; Scalmani, G.; Barone, V.; Petersson, G. A.; Nakatsuji, H.; Li, X.; Caricato, M.; Marenich, A. V.; Bloino, J.; Janesko, B. G.; Gomperts, R.; Mennucci, B.; Hratchian, H. P.; Ortiz, J. V.; Izmaylov, A. F.; Sonnenberg, J. L.; Williams-Young, D.; Ding, F.; Lipparini, F.; Egidi, F.; Goings, J.; Peng, B.; Petrone, A.; Henderson, T.; Ranasinghe, D.; Zakrzewski, V. G.; Gao, J.; Rega, N.; Zheng, G.; 
Liang, W.; Hada, M.; Ehara, M.; Toyota, K.; Fukuda, R.; Hasegawa, J.; Ishida, M.; Nakajima, T.; Honda, Y.; Kitao, O.; Nakai, H.; Vreven, T.; Throssell, K.; Montgomery, Jr., J. A.; Peralta, J. E.; Ogliaro, F.; Bearpark, M. J.; Heyd, J. J.; Brothers, E. N.; Kudin, K. N.; Staroverov, V. N.; Keith, T. A.; Kobayashi, R.; Normand, J.; Raghavachari, K.; Rendell, A. P.; Burant, J. C.; Iyengar, S. S.; Tomasi, J.; Cossi, M.; Millam, J. M.; Klene, M.; Adamo, C.; Cammi, R.; Ochterski, J. W.; Martin, R. L.; Morokuma, K.; Farkas, O.; Foresman, J. B.; Fox, D. J. Gaussian, Inc., Wallingford CT, 2016.

${ }^{60}$ Neese, F. The ORCA program system. WIREs Comput Mol Sci 2012, 2 (1), 73-78 DOI: $10.1002 /$ wcms.81.

${ }^{61}$ Neese, F. Software update: the ORCA program system, version 4.0. WIREs Comput Mol Sci 2018, 8 (1), e1327 DOI: $10.1002 /$ wcms.1327.

${ }^{62}$ Grochala, W.; Hoffmann, R.; Feng, J.; Ashcroft, N. W. The Chemical Imagination at Work InVery Tight Places. Angewandte Chemie International Edition 2007, 46 (20), 3620-3642. https://doi.org/10.1002/anie.200602485.

63 Pierotti, R. A. A Scaled Particle Theory of Aqueous and Nonaqueous Solutions. Chemical Reviews 1976, 76 (6), 717-726. https://doi.org/10.1021/cr60304a002.

${ }^{64}$ Hoffmann, R.; Woodward, R. B. Orbital Symmetries and Endo-Exo Relationships in Concerted Cycloaddition Reactions. J Am Chem Soc 1965, 87 (19), 4388-4389. https://doi.org/10.1021/ja00947a033.

65 (1) Yu, P.; Patel, A.; Houk, K. N. Transannular [6 + 4] and Ambimodal Cycloaddition in the Biosynthesis of Heronamide A. J Am Chem Soc 2015, 137 (42), 13518-13523. https://doi.org/10.1021/jacs.5b06656.

${ }^{66}$ (1) Yu, P.; Chen, T. Q.; Yang, Z.; He, C. Q.; Patel, A.; Lam, Y.; Liu, C.-Y.; Houk, K. N. Mechanisms and Origins of Periselectivity of the Ambimodal [6 + 4] Cycloadditions of Tropone to Dimethylfulvene. J Am Chem Soc 2017, 139 (24), 8251-8258. https://doi.org/10.1021/jacs.7b02966.

67 (1) Chen, S.; Yu, P.; Houk, K. N. Ambimodal Dipolar/Diels-Alder Cycloaddition Transition States Involving Proton Transfers. J Am Chem Soc 2018, 140 (51), 18124-18131. https://doi.org/10.1021/jacs.8b11080. 
68 (1) Chen, S.; Yu, P.; Houk, K. N. Ambimodal Dipolar/Diels-Alder Cycloaddition Transition States Involving Proton Transfers. J Am Chem Soc 2018, 140 (51), 18124-18131. https://doi.org/10.1021/jacs.8b11080.

${ }^{69}$ (1) Liu, F.; Chen, Y.; Houk, K. N. Huisgen's 1,3-Dipolar Cycloadditions to Fulvenes Proceed via Ambimodal [6+4]/[4+2] Transition States. Angewandte Chemie Int Ed 2020, 59 (30), 12412-12416. https://doi.org/10.1002/anie.202005265.

${ }^{70}$ (1) Jamieson, C. S.; Sengupta, A.; Houk, K. N. Cycloadditions of Cyclopentadiene and Cycloheptatriene with Tropones: All Endo-[6+4] Cycloadditions Are Ambimodal. J Am Chem Soc 2021, 143 (10), 3918-3926. https://doi.org/10.1021/jacs.0c13401.

${ }^{71}$ (1) Zhang, H.; Thøgersen, M. K.; Jamieson, C. S.; Xue, X.-S.; Jørgensen, K. A.; Houk, K. N. Ambimodal Transition States in Diels-Alder Cycloadditions of Tropolone and Tropolonate with N-Methylmaleimide. Angewandte Chemie Int Ed 2021. https://doi.org/10.1002/anie.202109608.

${ }^{72}$ Yang, T.; Fukuda, R.; Cammi, R.; Ehara, M. Diels-Alder Cycloaddition of Cyclopentadiene and C60 at the Extreme High Pressure. J Phys Chem 2017, 121 (22), 4363-4371. https://doi.org/10.1021/acs.jpca.7b02805.

${ }^{73}$ Chen, B.; Crespi, V.; Hoffmann, R. Theoretical Studies of Furan and Thiophene Nanothreads: Structures, Cycloaddition Barriers and Activation Volumes. ChemRxiv 2021. https://doi.org/10.26434/chemrxiv.14347163.v2. This content is a preprint and has not been peer-reviewed.

${ }^{74}$ Hammond, G. S. A Correlation of Reaction Rates. J Am Chem Soc 1955, 77 (2), 334-338. https://doi.org/10.1021/ja01607a027. 